\title{
Diffusion Tensor Imaging Parameters in Mild Traumatic Brain Injury and Its Correlation with Early Neuropsychological Impairment: A Longitudinal Study
}

\author{
Vigneswaran Veeramuthu, Vairavan Narayanan,, Tan Li Kuo,, Lisa Delano-Wood, ${ }^{3,4}$ Karuthan Chinna, ${ }^{5}$ \\ Mark William Bondi, ${ }^{3,4}$ Vicknes Waran, Dharmendra Ganesan,, and Norlisah Ramli²
}

\begin{abstract}
We explored the prognostic value of diffusion tensor imaging (DTI) parameters of selected white matter (WM) tracts in predicting neuropsychological outcome, both at baseline and 6 months later, among well-characterized patients diagnosed with mild traumatic brain injury (mTBI). Sixty-one patients with $\mathrm{mTBI}$ (mean age $=27.08$; standard deviation $[\mathrm{SD}], 8.55$ ) underwent scanning at an average of $10 \mathrm{~h}$ (SD, 4.26) post-trauma along with assessment of their neuropsychological performance at an average of $4.35 \mathrm{~h}$ (SD, 7.08) upon full Glasgow Coma Scale recovery. Results were then compared to 19 healthy control participants (mean age $=29.05$; SD, 5.84), both in the acute stage and 6 months post-trauma. DTI and neuropsychological measures between acute and chronic phases were compared, and significant differences emerged. Specifically, chronic-phase fractional anisotropy and radial diffusivity values showed significant group differences in the corona radiata, anterior limb of internal capsule, cingulum, superior longitudinal fasciculus, optic radiation, and genu of corpus callosum. Findings also demonstrated associations between DTI indices and neuropsychological outcome across two time points. Our results provide new evidence for the use of DTI as an imaging biomarker and indicator of WM damage occurring in the context of mTBI, and they underscore the dynamic nature of brain injury and possible biological basis of chronic neurocognitive alterations.
\end{abstract}

Key words: DTI; imaging biomarker; mTBI; neuropsychology; ROI; TBSS

\section{Introduction}

$\mathbf{M}$ LD TRAUMATIC BRAIN INJURY (MTBI) constitutes approximately $75-85 \%$ of all brain trauma cases. ${ }^{1}$ The longterm outcome of mTBI, however, is not well characterized owing to its considerable heterogeneity. One difficulty in accurately diagnosing mild neurotrauma relates to the frequent lack of radiological evidence to support the diagnosis, which often leads clinicians to diagnose mTBI based on clinical or cognitive symptoms known to overlap with other clinical conditions ${ }^{2}$ (e.g., hypoglycemic or vasovagal attacks and certain subtypes of mood disorders). Among the neuropsychological alterations that are commonly reported in patients with mTBI include impairment in attention, memory, psychomotor speed, and executive functions.
Mild neurotrauma is associated with traumatic axonal injury (TAI), which is described as a progressive event gradually evolving from focal axonal alteration to delayed axonal disconnection. ${ }^{3}$ Importantly, TAI is thought to represent one of the more common injuries observed in the aftermath of $\mathrm{mTBI}^{2}$ These subtle alterations of brain tracts or fiber pathways have been visualized using diffusion tensor imaging (DTI), which enables better visualization of the extent of early microstructural changes post-mTBI. ${ }^{2,4-6} \mathrm{~A}$ variety of metrics can be generated through DTI scans, including fractional anisotrophy (FA), which is a per-voxel indication of the directionality of underlying water diffusion. FA values range from 0 to 1 , where $\mathrm{FA}=0$ would indicate nondirectional diffusion (completely isotropic) and $\mathrm{FA}=1$ would indicate a single direction of diffusion where the water molecules are restricted to diffusion only

\footnotetext{
${ }^{1}$ Division of Neurosurgery, Department of Surgery, University of Malaya, Kuala Lumpur, Malaysia.

${ }^{2}$ University Malaya Research Imaging Center, University of Malaya, Kuala Lumpur, Malaysia.

${ }^{3}$ VA San Diego Healthcare System, San Diego, California.

${ }^{4}$ Department of Psychiatry, University of California San Diego, San Diego, California.

${ }^{5}$ Julius Center University Malaya, Department of Social and Preventive Medicine, University of Malaya, Kuala Lumpur, Malaysia.
}

(c) Vigneswaran Veeramuthu, Vairavan Narayanan, Tan Li Kuo, Lisa Delano-Wood, Karuthan Chinna, Mark William Bondi, Vicknes Waran, Dharmendra Ganesan, Norlisah Ramli; 2015; Published by Mary Ann Liebert, Inc. This Open Access article is distributed under the terms of the Creative Commons Attribution Noncommercial License (http://creativecommons.org/licenses/by-nc/4.0/) which permits any noncommercial use, distribution, and reproduction in any medium, provided the original author(s) and the source are credited. 
along a single axis (completely anisotropic). ${ }^{2,7}$ Reduced FA in the white matter (WM) is believed to reflect a loss of integrity, indicating possible damage to myelin or the axon membrane, reduced axonal packing, and/or decreased axonal coherence. ${ }^{2,8}$ Mean diffusivity (MD), on the other hand, describes the per-voxel average magnitude of water diffusion, regardless of diffusion direction. Differences in MD are thought to reflect overall restrictions to the movement of water diffusion, examples being the variations within the intra- and extracellular space. ${ }^{9}$ Radial diffusivity (RD) is defined as the diffusion of water perpendicular to WM fibers, ${ }^{10}$ which increases in response to demyelination ${ }^{11}$ and dysmyelination. ${ }^{12}$ Changes in axonal diameter or density can also influence changes observed in RD. ${ }^{13} \mathrm{FA}$ and MD values are usually inversely correlated with one another, given that myelination, which enforces directionality (thus increasing FA), also represents a restriction to overall movement (thus lowering MD). ${ }^{14,15}$ The demyelinating changes, as evinced by the changing $\mathrm{RD}$, however, are not expected to occur in the first week post-mTBI, despite the presence of axonal swelling and synaptic disruption. ${ }^{16}$

In mTBI, widespread changes in FA are frequently observed, especially in the frontal, mid-line, and temporal regions. Studies have shown that these changes can be detected as early as a few days to weeks after neurotrauma, as well as months or even a year after the initial insult. ${ }^{14,15,17}$ The shearing forces of trauma can breach the vascular permeability of vessels (hence rupturing them), sever fibers, and lyse cells, which deregulates the normal homeostasis of the blood-brain barrier, and usually manifests as cerebral vasogenic and cytotoxic edema. ${ }^{18}$ Evaluation of DTI indices enables the differentiation of these edemas, ${ }^{19}$ which is crucial to predicting long-term neurological outcomes, including neuropsychological performance (NP). Vasogenic edema commonly observed in $\mathrm{mTBI}$ is characterized by reduced FA, increased MD, and $\mathrm{RD}$ and is considered reversible. In contrast, cytotoxic edema, characterized by an increased FA, reduced MD, and RD, is considered irreversible and therefore confers a poor prognosis. ${ }^{2,19,20}$

To date, there are few studies that have been conducted longitudinally with acute mTBI samples to help elucidate the evolution of these DTI-based changes in mTBI over time. ${ }^{21-25}$ Unfortunately, existing studies have yielded generally equivocal findings. ${ }^{2,15,26-29}$ For example, some studies have reported decreased integrity of several tracts at different time intervals (acute and chronic), ${ }^{30-35}$ although others report elevated FA and reduced MD in the acute stage. ${ }^{16,21,36}$ Given that these findings may be inconsistent owing to various methodological differences, including patient recruitment, imaging protocol differences, varying intervals studied, samplesize differences, and heterogeneity of injury severities, we aimed to clarify these longitudinal DTI changes using a whole-brain WM measurement strategy with tract-based spatial statistics (TBSS) ${ }^{37}$ in mTBI and control groups. From the TBSS WM skeleton of comparison across subjects, we identified significant tract changes and correlated these regions with neuropsychological performances, both at admission and 6 months postinjury, in patients with mTBI. We also examined the relationship between anatomical correlates of tracts and cognition in an effort to improve prognostic values of DTI parameters in mTBI care.

\section{Methods}

\section{Participants}

Sixty-one patients with mTBI who presented to the emergency department (ED) of University of Malaya Medical Center (Kuala Lumpur, Malaysia) for a consecutive 11-month period between April 1, 2013 and March 1, 2014 were prospectively recruited for

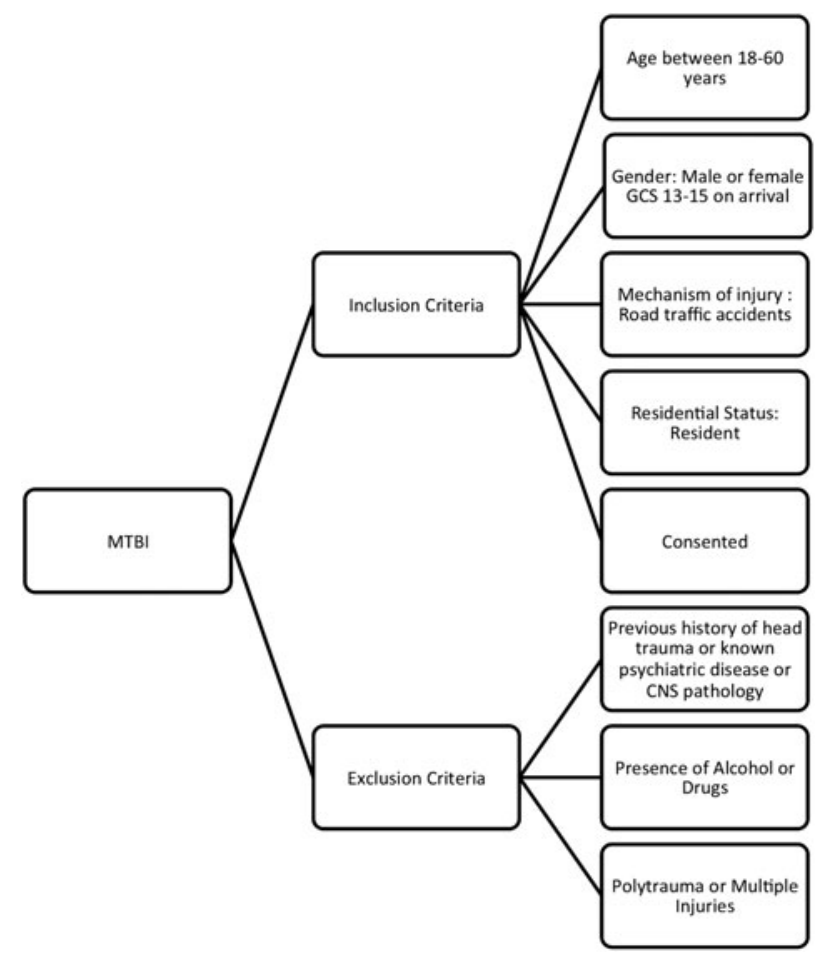

FIG. 1. Inclusion and exclusion criteria for patients with mTBI. mTBI, mild traumatic brain injury; GCS, Glasgow Coma Scale; CNS, central nervous system.

this study. Patients were selected based on the inclusion and exclusion criteria as presented in Figure 1. For the purposes of this study, mTBI was defined as acute TBI, consisting of nonpenetrating head trauma resulting in one or more of the following: confusion/disorientation; loss of consciousness (LOC) less than $30 \mathrm{~min}$; post-traumatic amnesia (PTA; less than $24 \mathrm{~h}$ in duration) and/or transient focal neurological signs or seizures; and Glasgow Coma Scale (GCS) of 13-15 upon acute clinical evaluation. The flow of the study is presented in Figure 2. Nineteen healthy age-matched control participants were also recruited for this study.

All subjects meeting criteria for the study underwent computed tomography (CT) scans of the brain in the ED using a Siemens Somatom Sensation 16 CT scanner (Siemens AG, Berlin, Germany). Cross-sectional images of the brain were obtained craniocaudally from the base of skull to vertex. Scan parameters used were $120 \mathrm{kVp}$, $300 \mathrm{mAs}$, and collimation of $16 \times 0.75 \mathrm{~mm}$ with standard brain and bone windowing. A neuroradiologist (N.R.) and a neurosurgeon (V.N.) who were blinded to the clinical diagnosis independently evaluated the images for each patient, and only patients who were deemed not requiring surgical intervention were included in this study. All subjects gave informed consent as required by the institutional research ethics committee and the hospital ethics committee (UM/EC Ref: 947.15)

\section{Study protocols}

Magnetic resonance imaging (MRI) and neuropsychological assessments were performed at admission and repeated again at 6 months post-trauma (Fig. 2). Healthy control participants were subjected to the same protocols as patients upon admission (i.e., MRI and neuropsychological assessment).

\section{Magnetic resonance imaging data acquisition}

All consented subjects were imaged on a 3T MRI scanner (Signa HDx; General Electric, Harvey, IL) using an eight-channel head 


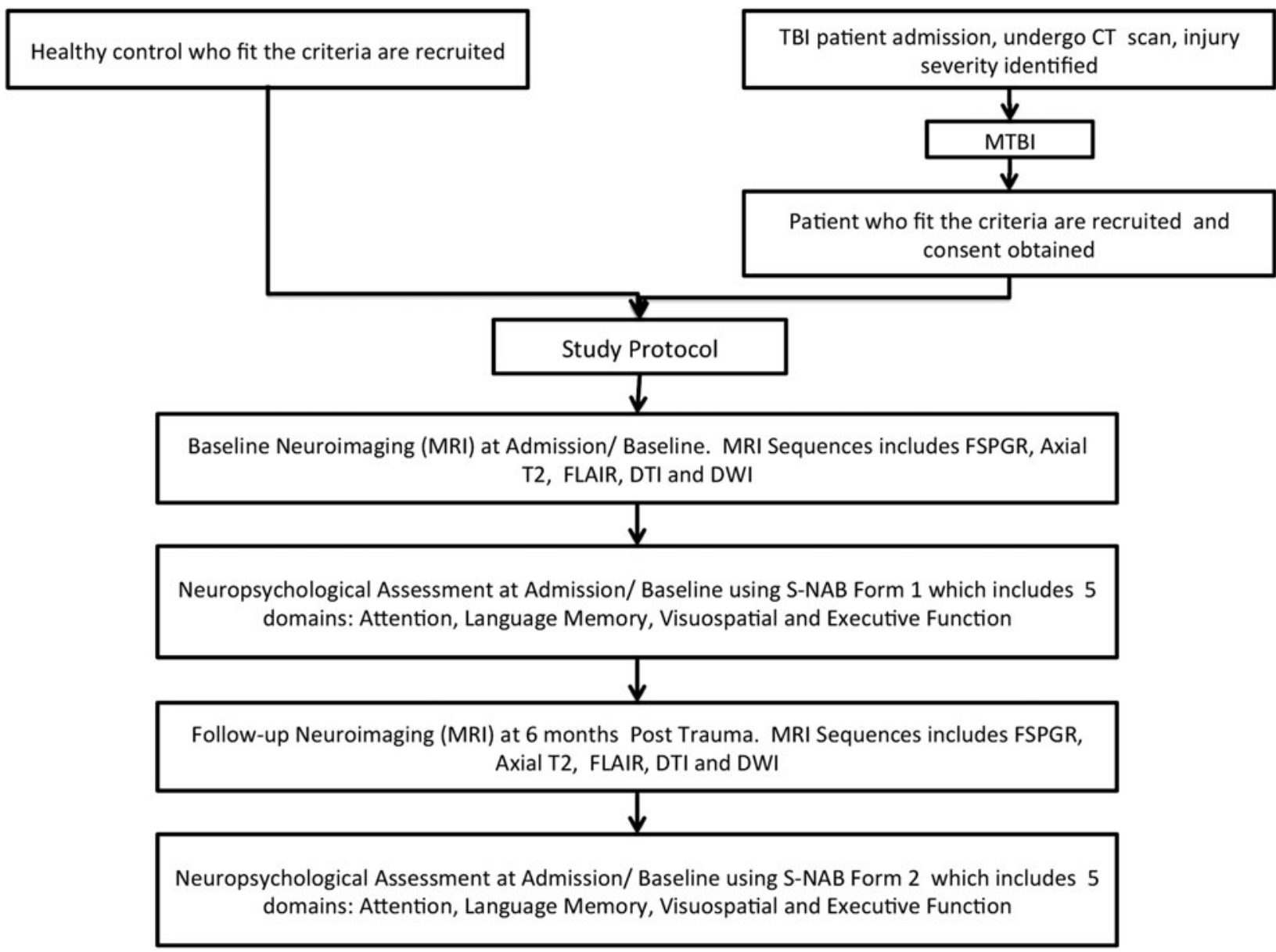

FIG. 2. Complete flowchart of the study protocols, patient and healthy control recruitment, and neuroimaging and neuropsychological assessments. TBI, traumatic brain injury; CT, computed tomography; mTBI, mild traumatic brain injury; MRI, magnetic resonance imaging; FLAIR, fluid-attentuated inversion recovery; DTI, diffusion tensor imaging; DWI, diffusion-weighted imaging; FSPGR, fast spoiled gradient echo; S-NAB, Neuropsychological Assessment Battery-Screening.

coil. The imaging protocol included: 1) axial T1-weighted threedimensional fast spoiled gradient echo (FSPGR; repetition time $[\mathrm{TR}]=$ minimum $6.7 \mathrm{~ms}$; echo time $[\mathrm{TE}]=$ minimum $1.9 \mathrm{~ms}$; field of view $[\mathrm{FOV}]=31 \mathrm{~mm}$; matrix $=256 \times 256$; slice thickness $=1.2 \mathrm{~mm}$; slice overlap $=0.6 \mathrm{~mm}$ with image scan time of $3 \mathrm{~min} 48 \mathrm{sec}$ ); 2) axial T2-weighted fast spin echo; TR $=4240 \mathrm{~ms} ; \mathrm{TE}=102 \mathrm{~ms} ; \mathrm{FOV}=24$ $\mathrm{mm}$; matrix $=512 \times 384$; thickness $=5 \mathrm{~mm}$; spacing $=1.5 \mathrm{~mm}$; and image scan time of $2 \mathrm{~min} 30 \mathrm{sec}$; and 3) coronal gradient echo $(\mathrm{TR}=655 \mathrm{~ms} ; \mathrm{TE}=20 \mathrm{~ms} ;$ flip angle $=15$ degrees; bandwidth $=31.25$; FOV $=24 \mathrm{~cm}$; matrix $=320 \times 256$; thickness $=5.0 \mathrm{~m}$; spacing $=1.5 \mathrm{~mm}$; and image scan time of $2 \mathrm{~min} 7 \mathrm{sec}$. The DTI sequence was obtained using these parameters: $\mathrm{TR}=13,000 \mathrm{~ms} ; \mathrm{TE}=81.2 \mathrm{~ms} ; \mathrm{FOV}=24 \mathrm{~mm}$; matrix $=128 \times 128$; slice thickness $=3.0 \mathrm{~mm} ; 32$ directions; diffusionweighted factor, $b=700 \mathrm{~s} / \mathrm{mm}^{2}$; and image scan time of $7 \mathrm{~min} 22 \mathrm{sec}$.

\section{Magnetic resonance imaging analysis}

Tract-based spatial statistics. Voxel-wise statistical analysis of the diffusion-weighted data was carried out using TBSS, ${ }^{37}$ part of the FSL (v5.0.6; University of Oxford, Oxford UK) software package. Initial preprocessing involved corrections for head movement and eddy currents, brain tissue extraction, and fitting of the diffusion tensor model. These were carried out using the FSL eddy_correct, bet, and dtifit tools, respectively. The standard TBSS analysis workflow was followed (V 1.2; http://fsl.fmrib.ox.ac.uk/ fsl/fslwiki/TBSS/UserGuide), with the following specific options: nonlinear registration to the FMRIB58_FA standard-space image; generation of a study-specific mean FA image for skeletonization; and a 0.2 threshold for the mean FA skeleton.

The voxel-wise statistical analysis was carried out using the FSL randomize tool. A two-group unpaired $t$-test design was used for comparing admission scans of control and mTBI subjects. A paired $t$-test design was used for comparing admission and follow-up scans of mTBI subjects. These statistical analyses were carried out separately for FA, MD, and RD values. In all cases, cluster-based thresholding was used, and 0.05 was adopted as the threshold for significant clusters.

Region of interest. In addition, we obtained mean FA, MD, and RD for all tracts identified on TBSS using region of interest (ROI) analysis. The image-processing pipeline consisted of preprocessing, image registration, and analysis, utilizing the FSL (v5.0.6; University of Oxford) and AFNI (v2011_12_21_1014; National Institute of Mental Health, Bethesda, MD) software packages. Initial preprocessing involved corrections for head movement and eddy currents, brain tissue extraction, and fitting of the diffusion tensor model. These were carried out using the FSL eddy_correct, bet, and dtifit tools, respectively. For image registration, the FSL tool fnirt was used to carry out nonlinear spatial registration of each subject to the FMRIB58_FA standard-space image, using the built-in FA_2_FMRIB58_1mm config file. This was assumed to be spatially compatible with the International Consortium of Brain Mapping (ICBM) DTI-81 atlas. Postregistration, composite axial slice images of the underlying FA and 
Table 1. List of S-NAB Module Subtests and Areas of Cognitive Domains Assessed

Neuropsychological assessment (S-NAB Form 1 and Form 2)

\begin{tabular}{ll}
\hline List of S-NAB module tests & Domains assessed \\
\hline Screening Orientation & Orientation \\
Screening Digits Forward & Attention \\
Screening Digits Backward & $\begin{array}{c}\text { Attention/Working } \\
\text { Memory }\end{array}$ \\
Screening Numbering and Letters & Attention \\
Screening Shape Learning Immediate & Memory \\
$\quad$ Recognition & \\
Screening Story Immediate Recall & Memory \\
Screening Delayed Shape Learning & Memory \\
$\quad$ Delayed Recognition & \\
Screening Story Learning Delayed & Memory \\
$\quad$ Recall & \\
Screening Naming & Language \\
Screening Auditory Comprehension & Language \\
$\quad$ three subtests) & \\
Screening Design Construction & Visuospatial \\
Screening Visual Discrimination & Visuospatial \\
Screening Word Generation & Executive Function/ \\
& Verbal Fluency \\
Mazes & Executive Function \\
\hline
\end{tabular}

S-NAB, Neuropsychological Assessment Battery-Screening.

ICBM atlas tract outlines were generated at $\mathrm{z}=42$ and $\mathrm{z}=82$ for each subject. An experienced neuroradiologist (N.R.) verified the overall registration quality of these images. Finally, the AFNI $3 \mathrm{dROIstats}$ tool was used to map the predefined ROIs to each individual subject and calculate the median FA, MD, and RD values for each tract.

\section{Neuropsychological assessment}

All subjects underwent cognitive assessment using the Screening Module of the Neuropsychological Assessment Battery (SNAB Form 1), which was performed by the neuropsychologist once the patient had recovered to a GCS score of 15 , which occurred with an average turnaround time of $4.35 \mathrm{~h}(\mathrm{SD}, 7.08)$ between time of trauma and full GCS recovery. The S-NAB comprises a comprehensive set of neuropsychological tests (refer to Table 1), with demographically corrected norms for adults between the ages of 18 and 97 years, assessing orientation and five cognitive domains (i.e., attention, memory, language, and visuospatial and executive functions). This battery consists of 12 individual tests across the five domains aforementioned. From these 12 tests, a total of $16 \mathrm{~T}$ scores are derived, 14 of which contribute toward five separate Screening Index (domain-specific) scores and one Total Screening Index score. ${ }^{38}$ The same subtests were repeated at 6 months by the same neuropsychologist using the S-NAB Form 2 in order to minimize practice effects.

\section{Statistical analysis}

An independent-samples $t$-test was used to establish whether mean values of FA, MD, and RD of selected WM tracts were significantly different between healthy control and mTBI groups during the acute phase (or baseline exam). The same test was also used to investigate whether patients (at admission) performed differently from healthy control participants on the neuropsychological assessment. A paired $t$-test was used thereafter to ascertain how the WM tracts had changed over time. TBSS skelotonized image of the significant changes observed over time were processed to better visualize any signifcant changes. Spearman's rho correlation coefficient was used to examine the association between WM ROIs (nine selected tracts) and NP over the different phases. Last, a simple frequency analysis of neuropsychological performance at 6 months post-trauma was performed to determine the types of changes observed in neuropsychological status longitudinally (improved, unchanged, or worsened).

\section{Results}

\section{Demographic and clinical data}

The demographic characteristics of study patients and healthy controls are presented in Table 2. The TBI group ranged in age between 18 and 53 years (mean, 27.08; standard deviation [SD], $8.55)$, was predominantly male $(88.5 \%)$, and had a mean age of 29.05 (SD, 5.84). There was no significant difference in the mean

Table 2. Demographics and Clinical Data of Patients and Healthy Controls at Admission/Baseline

\begin{tabular}{|c|c|c|c|c|c|c|c|c|}
\hline & \multicolumn{4}{|c|}{$T B I(\mathrm{n}=61)$} & \multicolumn{4}{|c|}{ Healthy control $(\mathrm{n}=19)$} \\
\hline & Median & Mean & $S D$ & Range & Median & Mean & $S D$ & Range \\
\hline Age & 24.0 & 27.08 & 8.55 & $18-53$ & 29.0 & 29.05 & 5.83 & $18-43$ \\
\hline Education (years) & 11.0 & 11.52 & 1.94 & $6-19$ & 11.0 & 15.95 & 2.01 & $11-20$ \\
\hline Time to scan (hours/months) & 9.0 & 10.01 & 4.26 & $0-23$ & $\mathrm{n} / \mathrm{a}$ & $\mathrm{n} / \mathrm{a}$ & $\mathrm{n} / \mathrm{a}$ & $\mathrm{n} / \mathrm{a}$ \\
\hline GCS & 15.0 & 14.44 & 0.74 & $13-15$ & 15 & 15 & 0 & 0 \\
\hline Time to full GCS (hours) & 0.5 & 4.35 & 7.08 & $0-23$ & $\mathrm{n} / \mathrm{a}$ & $\mathrm{n} / \mathrm{a}$ & $\mathrm{n} / \mathrm{a}$ & $\mathrm{n} / \mathrm{a}$ \\
\hline LOC (\%) & 77.0 & & & & $\mathrm{n} / \mathrm{a}$ & & & \\
\hline PTA $(\%)$ & 73.8 & & & & $\mathrm{n} / \mathrm{a}$ & & & \\
\hline Gender ( $\%$ male $)$ & 88.5 & & & & 78.9 & & & \\
\hline Ethnicity (\% Polynesian Malay) & 73.8 & & & & 57.9 & & & \\
\hline Handedness (\% right-handed) & 86.9 & & & & 89.5 & & & \\
\hline GOSE 8 at discharge $(\%)$ & 88.5 & & & & $\mathrm{n} / \mathrm{a}$ & & & \\
\hline GOSE 8 at follow-up (\%) & $\mathrm{n} / \mathrm{a}$ & & & & $\mathrm{n} / \mathrm{a}$ & & & \\
\hline \multicolumn{9}{|l|}{ Types of MVAs involved: } \\
\hline -Motorcycle vs. car (\%) & 49.2 & & & & $\mathrm{n} / \mathrm{a}$ & & & \\
\hline -Motorcycle vs. motorcycle (\%) & 32.8 & & & & $\mathrm{n} / \mathrm{a}$ & & & \\
\hline -Others $(\%)$ & 18.00 & & & & $\mathrm{n} / \mathrm{a}$ & & & \\
\hline
\end{tabular}

GCS, Glasgow Coma Scale; LOC, loss of consciousness; PTA, post-traumatic amnesia; GOSE, Glasgow Outcome Score Extended; MVAs, motor vehicle accidents; TBI, traumatic brain injury; SD, standard deviation; n/a, not applicable. 
age of healthy controls versus the TBI patient group $\left(t_{(78)}=0.937\right.$; $p=0.256$ ). However, the mean years of education differed significantly between groups (TBI, 11.52 [SD, 1.94] vs. controls: 15.95 [SD, 2.01] years). Admission GCS scores of patients were within the range of 13-15 (mean $=14.44$; SD, 0.74). Seventy-seven percent of patients had experienced transient LOC whereas $73.8 \%$ reported PTA. Average time to scan of patients during the acute phase was $10.01 \mathrm{~h}$ (SD, 4.26), whereas the chronic phase scan was performed at 6 months postinjury (mean $=6.05$; SD, 0.12). Of the 61 patients, 33 underwent the chronic phase repeat scans ( 3 patients were later dropped during the postscan processing because their scans had major artifacts in echo planar imaging). All healthy controls had no significant neurological findings.

\section{Diffusion metrics, intergroup differences,} and intragroup changes over time

Table 3 presents mean FA, MD, and RD values of both patients and healthy control participants during the acute phase. At baseline, the mTBI group showed significantly lower splenium FA $\left(t_{(78)}=2.196\right.$; $p=0.04)$, when compared to the control group, whereas the $\mathrm{MD}_{\mathrm{a}}$ value was significantly higher in the mTBI versus control group in the posterior limb of the internal capsule (PLIC; $\left(t_{(78)}=-2.179 ; p=0.03\right.$ ); cingulum $\left(\mathrm{CG} ; t_{(78)}=-2.29 ; p=0.02\right)$; optic radiation $\left(\mathrm{OR} ; t_{(78)}=\right.$ $-3.176 ; p=0.002)$; and splenium (SCC; $\left.t_{(78)}=-2.514 ; p=0.02\right)$. Group differences for the $\mathrm{RD}_{\mathrm{a}}$ values were observed only in the $\mathrm{OR}$ $\left(t_{(78)}=2.637 ; p=0.02\right)$ and SCC $\left(t_{(78)}=2.519 ; p=0.02\right)$. See Figure 3 for the TBSS skeletonized image reflecting the significant area of differences between the groups.

The paired $t$-test results (FA, MD, and RD) of patients' WM pathway changes, as observed at 6 months (chronic phase) against the baseline (acute phase) values, are presented in Table $4 \mathrm{a}, 4 \mathrm{~b}$, and $4 \mathrm{c}$, along with their effect sizes (Cohen's $d$ with $<0.2=$ small effect,
$>0.2<0.8=$ moderate effect, and $>0.8=$ large effect). Various differences were observed in all DTI parameters, with greater alterations noted in FA values across regions. Specifically, FA of the corona radiata $\left(\mathrm{CR} ; t_{(28)}=3.497 ; p=0.002 ; d=0.661\right)$, anterior limb of internal capsule (ALIC; $t_{(28)}=2.582 ; p=0.016 ; d=0.488$ ), $\mathrm{CG}(t(28)=2.973 ; p=0.006 ; d=0.562)$, superior longitudinal fasciculus (SLF; $\left.t_{(28)}=2.404 ; p=0.024 ; d=0.454\right)$, OR $\left(t_{(28)}=2.643\right.$; $p=0.014 ; d=0.499$ ), and the genu of corpus callosum (GCC; $\left.t_{(28)}=2.732 ; p=0.011 ; d=0.516\right)$ were significantly lower in the TBI group. Almost all of the MD values across the phases showed no significant changes, except the CG $\left(t_{(28)}=3.189 ; p=0.004 ; d=0.603\right)$, which was significantly higher in the TBI group. Although the changes in PLIC across the phases were found to be statistically nonsignificant $\left(t_{(28)}=1.494 ; p=0.15\right)$, the effect size, however, suggests a moderate level of change $(d=0.282)$. Finally, there were no significant differences in RD values across time points. However, Cohen's $d$ effect-size calculation indicated moderate effect sizes for changes in the RD of $\mathrm{CR}\left(t_{(28)}=1.582 ; p=0.126 ; d=0.299\right), \mathrm{CG}\left(t_{(28)}=1.54\right.$; $p=0.136 ; d=0.291), \mathrm{OR}\left(t_{(28)}=1.87 ; p=0.073 ; d=0.353\right)$, and GCC $(t(28)=1.975 ; p=0.059 ; d=0.373)$.

All the above-stated significant differences were reflected in the TBSS images of the skeletonized tracts as well (see Fig. 4).

\section{Neuropsychological performance}

Table 5 presents the mean interpretive categories score comparison for the domain-specific NP among mTBI and healthy control groups. During the acute phase, patients with mTBI performed poorly across all domains, in comparison to the healthy control group. The independent-samples $t$-tests of both groups and their NPs indicated that the mTBI group was significantly poorer (all $p$ values, $<0.001$ ) on all but one of the neuropsychological domains (visuospatial functions, $\left.t_{(78)}=0.055 ; p=0.956\right)$. Meanwhile, during the chronic phase,

Table 3. Differences of DTI Metrics (FA, MD, and RD) between Patients with mTBI Acutely and Baseline Scans of Healthy Controls

\begin{tabular}{|c|c|c|c|c|c|c|c|c|c|c|c|}
\hline \multirow[b]{2}{*}{ DTI metrics vs. tracts } & \multirow[b]{2}{*}{ Group } & \multirow[b]{2}{*}{$\mathrm{N}$} & \multicolumn{3}{|c|}{$F A$} & \multicolumn{3}{|c|}{$M D$} & \multicolumn{3}{|c|}{$R D$} \\
\hline & & & Mean & $S D$ & $\mathrm{p}$ value & Mean & $S D$ & $\mathrm{p}$ value & Mean & $S D$ & $\mathrm{p}$ value \\
\hline Middle cerebellar peduncle & $\begin{array}{l}\text { CTRL } \\
\text { TBI }\end{array}$ & $\begin{array}{l}19 \\
61\end{array}$ & $\begin{array}{l}0.611 \\
0.612\end{array}$ & $\begin{array}{l}0.032 \\
0.025\end{array}$ & 0.869 & $\begin{array}{l}0.698 \\
0.711\end{array}$ & $\begin{array}{l}0.034 \\
0.036\end{array}$ & 0.168 & $\begin{array}{l}0.432 \\
0.438\end{array}$ & $\begin{array}{l}0.038 \\
0.033\end{array}$ & 0.514 \\
\hline Corona radiata & $\begin{array}{l}\text { CTRL } \\
\text { TBI }\end{array}$ & $\begin{array}{l}19 \\
61\end{array}$ & $\begin{array}{l}0.532 \\
0.538\end{array}$ & $\begin{array}{l}0.028 \\
0.025\end{array}$ & 0.435 & $\begin{array}{l}0.792 \\
0.802\end{array}$ & $\begin{array}{l}0.019 \\
0.025\end{array}$ & 0.104 & $\begin{array}{l}0.535 \\
0.537\end{array}$ & $\begin{array}{l}0.029 \\
0.028\end{array}$ & 0.785 \\
\hline Anterior limb of internal capsule & $\begin{array}{l}\text { CTRL } \\
\text { TBI }\end{array}$ & $\begin{array}{l}19 \\
61\end{array}$ & $\begin{array}{l}0.636 \\
0.629\end{array}$ & $\begin{array}{l}0.024 \\
0.026\end{array}$ & 0.297 & $\begin{array}{l}0.791 \\
0.800\end{array}$ & $\begin{array}{l}0.018 \\
0.027\end{array}$ & 0.147 & $\begin{array}{l}0.462 \\
0.474\end{array}$ & $\begin{array}{l}0.023 \\
0.029\end{array}$ & 0.130 \\
\hline Posterior limb of internal capsule & $\begin{array}{l}\text { CTRL } \\
\text { TBI }\end{array}$ & $\begin{array}{l}19 \\
61\end{array}$ & $\begin{array}{l}0.719 \\
0.712\end{array}$ & $\begin{array}{l}0.028 \\
0.025\end{array}$ & 0.315 & $\begin{array}{l}0.784 \\
0.800\end{array}$ & $\begin{array}{l}0.028 \\
0.027\end{array}$ & 0.028 & $\begin{array}{l}0.392 \\
0.406\end{array}$ & $\begin{array}{l}0.031 \\
0.030\end{array}$ & 0.110 \\
\hline Cingulum & $\begin{array}{l}\text { CTRL } \\
\text { TBI }\end{array}$ & $\begin{array}{l}19 \\
61\end{array}$ & $\begin{array}{l}0.560 \\
0.555\end{array}$ & $\begin{array}{l}0.029 \\
0.030\end{array}$ & 0.561 & $\begin{array}{l}0.757 \\
0.777\end{array}$ & $\begin{array}{l}0.028 \\
0.032\end{array}$ & 0.020 & $\begin{array}{l}0.495 \\
0.510\end{array}$ & $\begin{array}{l}0.027 \\
0.032\end{array}$ & 0.064 \\
\hline Superior longitudinal fasiculus & $\begin{array}{l}\text { CTRL } \\
\text { TBI }\end{array}$ & $\begin{array}{l}19 \\
61\end{array}$ & $\begin{array}{l}0.522 \\
0.519\end{array}$ & $\begin{array}{l}0.024 \\
0.024\end{array}$ & 0.656 & $\begin{array}{l}0.769 \\
0.775\end{array}$ & $\begin{array}{l}0.022 \\
0.024\end{array}$ & 0.280 & $\begin{array}{l}0.528 \\
0.535\end{array}$ & $\begin{array}{l}0.028 \\
0.025\end{array}$ & 0.325 \\
\hline Optic radiation & $\begin{array}{l}\text { CTRL } \\
\text { TBI }\end{array}$ & $\begin{array}{l}19 \\
61\end{array}$ & $\begin{array}{l}0.631 \\
0.619\end{array}$ & $\begin{array}{l}0.036 \\
0.029\end{array}$ & 0.154 & $\begin{array}{l}0.848 \\
0.878\end{array}$ & $\begin{array}{l}0.039 \\
0.035\end{array}$ & 0.002 & $\begin{array}{l}0.494 \\
0.521\end{array}$ & $\begin{array}{l}0.048 \\
0.039\end{array}$ & 0.015 \\
\hline Genu of corpus callosum & $\begin{array}{l}\text { CTRL } \\
\text { TBI }\end{array}$ & $\begin{array}{l}19 \\
61\end{array}$ & $\begin{array}{l}0.766 \\
0.760\end{array}$ & $\begin{array}{l}0.030 \\
0.031\end{array}$ & 0.499 & $\begin{array}{l}0.811 \\
0.820\end{array}$ & $\begin{array}{l}0.027 \\
0.036\end{array}$ & 0.309 & $\begin{array}{l}0.360 \\
0.368\end{array}$ & $\begin{array}{l}0.039 \\
0.043\end{array}$ & 0.519 \\
\hline Splenium of corpus callosum & $\begin{array}{l}\text { CTRL } \\
\text { TBI }\end{array}$ & $\begin{array}{l}19 \\
61\end{array}$ & $\begin{array}{l}0.855 \\
0.842\end{array}$ & $\begin{array}{l}0.028 \\
0.023\end{array}$ & 0.038 & $\begin{array}{l}0.737 \\
0.757\end{array}$ & $\begin{array}{l}0.034 \\
0.030\end{array}$ & 0.016 & $\begin{array}{l}0.233 \\
0.258\end{array}$ & $\begin{array}{l}0.045 \\
0.037\end{array}$ & 0.020 \\
\hline
\end{tabular}

Variances in the group were similar for all comparisons

DTI, diffusion tensor imaging; FA, fractional anisotropy; MD, medial diffusivity; RD, radial diffusivity; mTBI, mild traumatic brain injury; SD, standard deviation; CTRL, control; TBI, traumatic brain injury. 

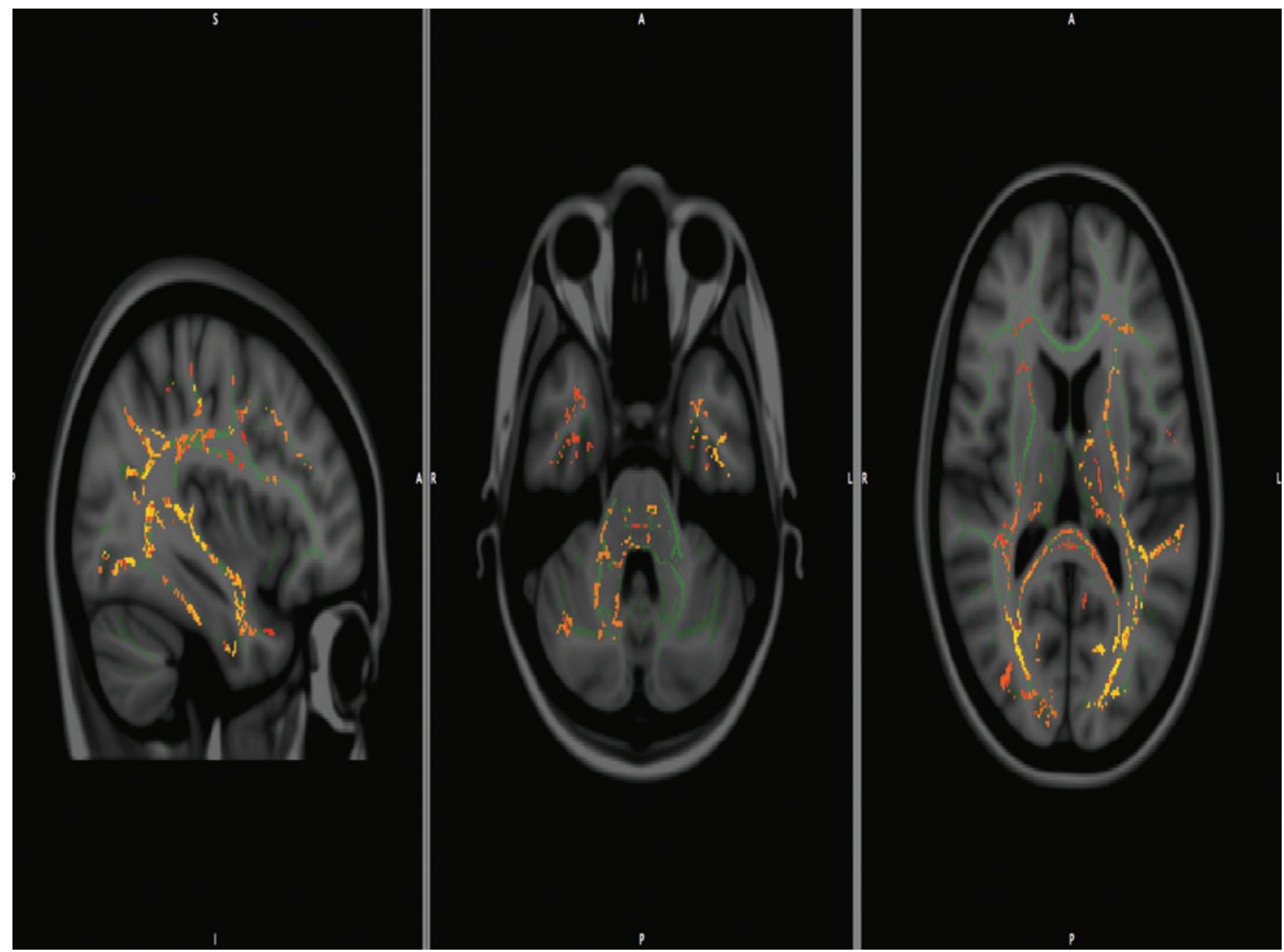

FIG. 3. Tract-based spatial statistics (TBSS) showing composite of fractional anisotropy/mean diffusivity/radial diffusivity (FA/MD/ $\mathrm{RD}$ ) voxels of the white matter tracts that showed significant difference between control and mild traumatic brain injury. (mTBI).

patients with mTBI continued to perform poorly across most domains, in exception of visuospatial functions, where the patients outperformed healthy controls $\left(t_{(48)}=-2.373 ; p=0.021\right)$.

\section{Associations between diffusion tensor imaging parameters and neuropsychological performance}

Longitudinal analysis of WM tract changes against the NP at different intervals among a subset of study patients $(n=30)$ are presented in Table 6. Most of the observed FA associations with neurocognitive status (acute and chronic) represented negative associations. $\mathrm{FA}_{\mathrm{a}}$ negatively correlated with the following acute $\mathrm{NP}_{\mathrm{a}}$ : attention versus $\mathrm{CR}(r=-0.429 ; p<0.05)$; language versus CR $(r=-0.375 ; p<0.05)$; language versus $\operatorname{SLF}(r=-0.557$; $p<0.01)$; and language versus GCC $(r=-0.443 ; p<0.05)$. Acute FAs $\left(\mathrm{FA}_{\mathrm{a}}\right)$ were also negatively correlated with $\mathrm{NP}_{\mathrm{c}}$, as follows: language versus middle cerebelar peduncle $\left(\mathrm{MCP} ; r_{s}=-0.440\right.$; $p<0.05)$; attention versus CR $\left(r_{s}=-0.441 ; p<0.05\right)$; language versus CR $\left(r_{s}=-0.415 ; p<0.05\right)$; attention versus $\operatorname{SLF}\left(r_{s}=\right.$ $-0.417 ; p<0.05)$; language versus SLF $\left(r_{s}=-0.409 ; p<0.05\right)$; spatial versus CG $\left(r_{s}=-0.489 ; p<0.05\right)$. The two positive correlations observed in the chronic phase were the spatial versus CG $\left(r_{s}=0.489 ; p<0.05\right)$ and spatial versus SCC $(r=0.402 ; p<0.05)$. The significantly reduced $\mathrm{FA}_{\mathrm{c}}$ had only two associations with the $\mathrm{NP}_{\mathrm{c}}$ : language versus $\mathrm{CR}(r=-0.400 ; p<0.05)$ and spatial versus $\operatorname{SCC}(r=0.402 ; p<0.05)$
$\mathrm{MD}_{\mathrm{a}}$ showed limited associations with the $\mathrm{NP}_{\mathrm{c}}$. The following were the only $\mathrm{MD}_{\mathrm{a}}$ values associated with the $\mathrm{NP}_{\mathrm{c}}$ : attention versus SLF $\left(r_{s}=0.404 ; p<0.05\right) ; \quad$ spatial versus CG $\left(r_{s}=-0.390\right.$; $p<0.05)$; and spatial versus GCC $\left(r_{s}=-0.404 ; p<0.05\right)$. No associations between the $\mathrm{MD}_{\mathrm{c}}$ and $\mathrm{NP}_{\mathrm{c}}$ were observed.

The RD of seven of nine tracts studied was significantly associated with attention, language, spatial, and executive function in both phases of the study. Specifically, $\mathrm{RD}_{\mathrm{a}}$ was associated with three $\mathrm{NP}_{\mathrm{a}}$ scores: attention versus $\mathrm{CR}(r=0.485 ; p<0.05)$, attention versus SLF $(r=0.487 ; p<0.05)$; and spatial versus OR $(r=-0.378 ; p<0.05)$. Additional associations were subsequently observed between $\mathrm{RD}_{\mathrm{a}}$ and $\mathrm{NP}_{\mathrm{c}}$, which were as follows: language versus MCP $(r=0.398 ; p<0.05)$; language versus CR $\left(r_{s}=0.529\right.$; $p<0.01)$; attention versus SLF $(r=0.450 ; p<0.05)$; language versus SLF $(r=0.491 ; p<0.05)$; language versus CG $(r=0.423$; $p<0.05)$; spatial versus CG $(r=0.626 ; p<0.01)$; and attention versus GCC $(r=-0.378 ; p<0.05)$. Increased $\mathrm{RD}_{\mathrm{c}}$ values were also associated with some domains of the $\mathrm{NP}_{\mathrm{c}}$, including language versus MCP $\left(r_{s}=0.438 ; p<0.05\right)$, executive function versus $\mathrm{CR}$ $\left(r_{s}=0.389 ; p<0.05\right)$; executive function versus CG $\left(r_{s}=-0.404\right.$; $p<0.05)$; and executive function versus SCC $\left(r_{s}=-0.391\right.$; $p<0.05)$. No association was found between the domains of memory and any of the WM tracts investigated in this study. We also found no associations between the DTI parameters (FA, MD, and RD) and any domains of the NP in the healthy control group (kindly refer to Table 6b). 
Table 4a. Paired $T$-Test Analysis of FA Changes (Acute vs. Chronic) in Tracts of Interest and Cohen's $D$ EfFect-Size Calculation

\begin{tabular}{|c|c|c|c|c|c|c|}
\hline & $\begin{array}{c}\text { Acute } \\
\text { Mean } \pm S D\end{array}$ & $\begin{array}{c}\text { Chronic } \\
\text { Mean } \pm S D\end{array}$ & $\mathrm{t}$ & df & $\mathrm{p}$ value & $\begin{array}{c}\text { Effect size } \\
\mathrm{d}\end{array}$ \\
\hline Middle cerebellar peduncle & $0.612 \pm 0.025$ & $0.607 \pm 0.020$ & 1.412 & 28 & 0.170 & 0.267 \\
\hline Corona radiata & $0.538 \pm 0.025$ & $0.531 \pm 0.027$ & 3.497 & 28 & 0.002 & 0.661 \\
\hline Anterior limb of internal capsule & $0.629 \pm 0.026$ & $0.627 \pm 0.025$ & 2.582 & 28 & 0.016 & 0.488 \\
\hline Posterior limb of internal capsule & $0.712 \pm 0.025$ & $0.710 \pm 0.028$ & 1.524 & 28 & 0.140 & 0.288 \\
\hline Cingulum & $0.555 \pm 0.030$ & $0.550 \pm 0.034$ & 2.973 & 28 & 0.006 & 0.562 \\
\hline Superior longitudinal fasiculus & $0.519 \pm 0.024$ & $0.516 \pm 0.022$ & 2.404 & 28 & 0.024 & 0.454 \\
\hline Optic radiation & $0.619 \pm 0.029$ & $0.614 \pm 0.034$ & 2.643 & 28 & 0.014 & 0.499 \\
\hline Genu of corpus callosum & $0.760 \pm 0.031$ & $0.749 \pm 0.034$ & 2.732 & 28 & 0.011 & 0.516 \\
\hline Splenium of corpus callosum & $0.842 \pm 0.023$ & $0.841 \pm 0.029$ & 0.307 & 28 & 0.762 & 0.058 \\
\hline
\end{tabular}

FA, fractional anisotropy; SD, standard deviation.

Table 4b. Paired $T$-Test Analysis of the MD Changes (Acute vs. Chronic) in Tracts of Interest and Cohen's $D$ Effect-Size Calculation

\begin{tabular}{|c|c|c|c|c|c|c|}
\hline & $\begin{array}{c}\text { Acute } \\
\text { Mean } \pm S D\end{array}$ & $\begin{array}{c}\text { Chronic } \\
\text { Mean } \pm S D\end{array}$ & $\mathrm{t}$ & df & $\mathrm{p}$ value & $\begin{array}{c}\text { Effect size } \\
\mathrm{d}\end{array}$ \\
\hline Middle cerebellar peduncle & $0.711 \pm 0.036$ & $0.714 \pm 0.025$ & 0.095 & 28 & 0.93 & 0.018 \\
\hline Corona radiata & $0.802 \pm 0.025$ & $0.805 \pm 0.024$ & 0.683 & 28 & 0.50 & 0.129 \\
\hline Anterior limb of internal capsule & $0.800 \pm 0.027$ & $0.797 \pm 0.020$ & 0.487 & 28 & 0.63 & 0.092 \\
\hline Posterior limb of internal capsule & $0.800 \pm 0.027$ & $0.793 \pm 0.019$ & 1.494 & 28 & 0.15 & 0.282 \\
\hline Cingulum & $0.777 \pm 0.032$ & $0.765 \pm 0.026$ & 3.189 & 28 & 0.00 & 0.603 \\
\hline Superior longitudinal fasiculus & $0.775 \pm 0.024$ & $0.773 \pm 0.022$ & 0.288 & 28 & 0.78 & 0.054 \\
\hline Optic radiation & $0.878 \pm 0.035$ & $0.880 \pm 0.036$ & 0.028 & 28 & 0.98 & 0.005 \\
\hline Genu of corpus callosum & $0.820 \pm 0.036$ & $0.828 \pm 0.031$ & 0.622 & 28 & 0.54 & 0.118 \\
\hline Splenium of corpus callosum & $0.757 \pm 0.030$ & $0.756 \pm 0.028$ & 0.262 & 28 & 0.80 & 0.050 \\
\hline
\end{tabular}

MD, medial diffusivity; SD, standard deviation.

Table 4C. Paired t-Test Table of RD Changes (Acute vs. Chronic) in Tracts of Interest and Cohen's $D$ EFFect-Size Calculation

\begin{tabular}{|c|c|c|c|c|c|c|}
\hline & $\begin{array}{c}\text { Acute } \\
\text { Mean } \pm S D\end{array}$ & $\begin{array}{c}\text { Chronic } \\
\text { Mean } \pm S D\end{array}$ & $\mathrm{t}$ & df & $\mathrm{p}$ value & $\begin{array}{c}\text { Effect size } \\
\mathrm{d}\end{array}$ \\
\hline Middle cerebellar peduncle & $0.438 \pm 0.033$ & $0.443 \pm 0.027$ & 0.427 & 28 & 0.673 & 0.081 \\
\hline Corona radiata & $0.537 \pm 0.028$ & $0.542 \pm 0.026$ & 1.582 & 28 & 0.126 & 0.299 \\
\hline Anterior limb of internal capsule & $0.474 \pm 0.029$ & $0.473 \pm 0.024$ & 0.818 & 28 & 0.421 & 0.155 \\
\hline Posterior limb of internal capsule & $0.406 \pm 0,030$ & $0.403 \pm 0.030$ & 0.049 & 28 & 0.961 & 0.009 \\
\hline Cingulum & $0.510 \pm 0.032$ & $0.504 \pm 0.030$ & 1.540 & 28 & 0.136 & 0.291 \\
\hline Superior longitudinal fasiculus & $0.535 \pm 0.025$ & $0.535 \pm 0.022$ & 1.429 & 28 & 0.165 & 0.270 \\
\hline Optic radiation & $0.521 \pm 0.039$ & $0.528 \pm 0.044$ & 1.870 & 28 & 0.073 & 0.353 \\
\hline Genu of corpus callosum & $0.368 \pm 0.043$ & $0.382 \pm 0.041$ & 1.975 & 28 & 0.059 & 0.373 \\
\hline Splenium of corpus callosum & $0.258 \pm 0.037$ & $0.258 \pm 0.043$ & 0.037 & 28 & 0.971 & 0.007 \\
\hline
\end{tabular}

$\mathrm{RD}$, radial diffusivity; $\mathrm{SD}$, standard deviation.

\section{Neuropsychological outcomes}

Table 7 presents the neuropsychological profiles of the subset of patients $(n=30)$ who had completed neuropsychological evaluations, as well as imaging, both at admission and at month 6 followup. Within the domain of attention, $56.7 \%$ of the patients remained impaired at 6 months post-trauma, 3.33\% worsened, and the remaining improved or remained unaffected. Within the domain of language function, $63.3 \%$ of patients remained impaired, $26.7 \%$ improved, $6.67 \%$ remained unaffected, and $3.33 \%$ worsened. Approximately $33.3 \%$ of patients remained impaired within the domain of memory 6 months post-trauma with essentially equivocal changes (26.7\% worsened, $26.7 \%$ remained unaffected, and $13.3 \%$ with improved memory status). The majority of patients $(53.3 \%)$ remained unaffected for spatial function, although $23.3 \%$ showed signs of delayed impairments in the chronic phase. A total of $70.0 \%$ of the subset patient group remained impaired with respect to executive functioning, with only $20.0 \%$ of these patients showing signs of improvement or recovery after the 6-month period posttrauma.

\section{Discussion}

We examined the relationship between microstructural changes and neuropsychological functioning that takes place in the immediate aftermath of an mTBI as well as 6 months post-TBI. Specifically, the initial neuropsychological assessment was completed, on average, $4.35 \mathrm{~h}$ after full GCS recovery, and the neuroimaging 

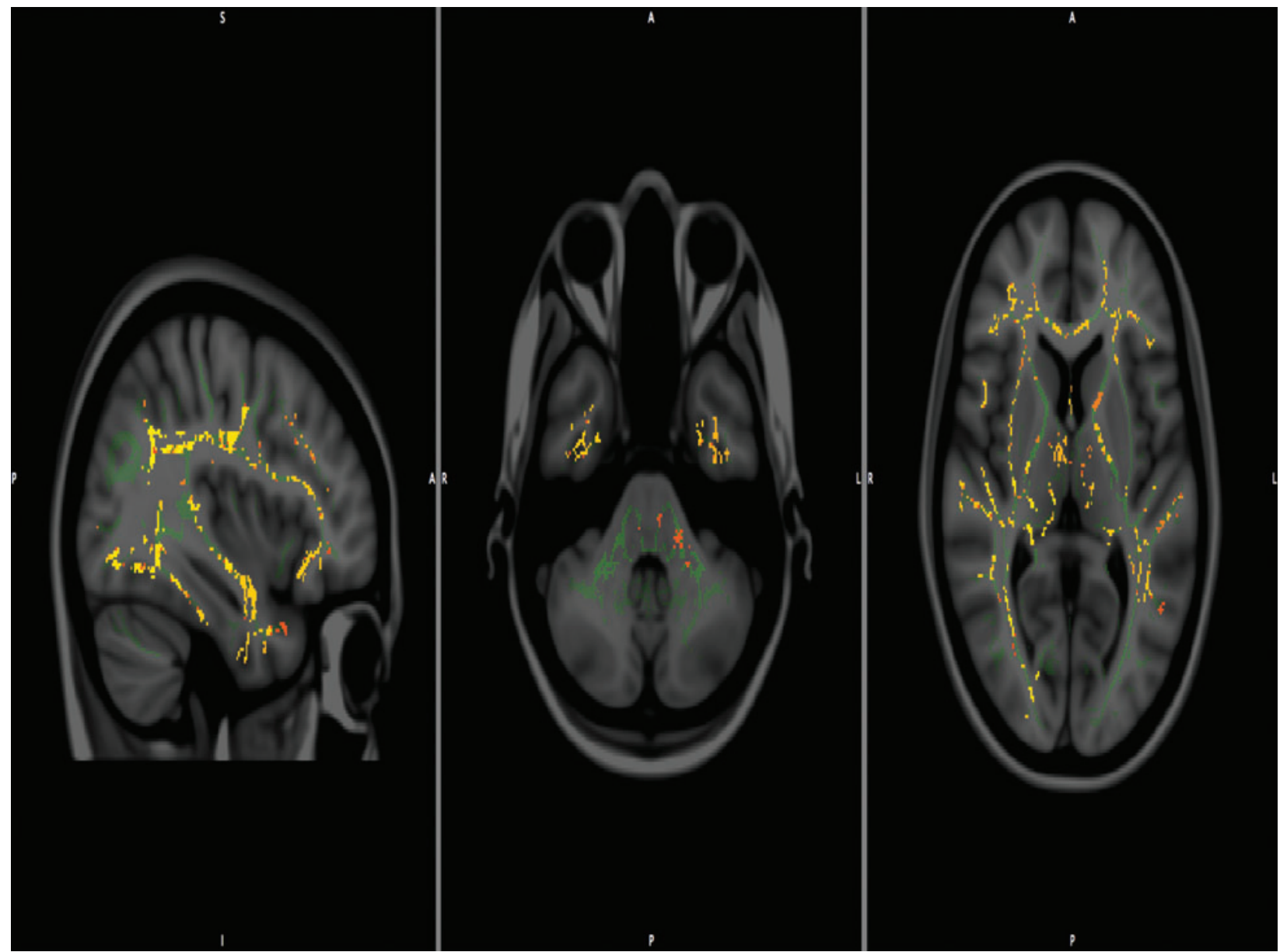

FIG. 4. Tract-based spatial statistics (TBSS) showing composite of fractional anisotropy/mean diffusivity/radial diffusivity (FA/MD/ $\mathrm{RD}$ ) voxels of the white matter tracts that showed significant different between acute and chronic phase (for patients with mild traumatic brain injury $[\mathrm{mTBI}])$.

Table 5. Mean of S-NAB Interpretive Categories Score at Acute and Chronic Phase of Both Patients and Controls and Intergroup Differences (IndePendent $T$-Test) in Domain-Specific Neuropsychological Performance

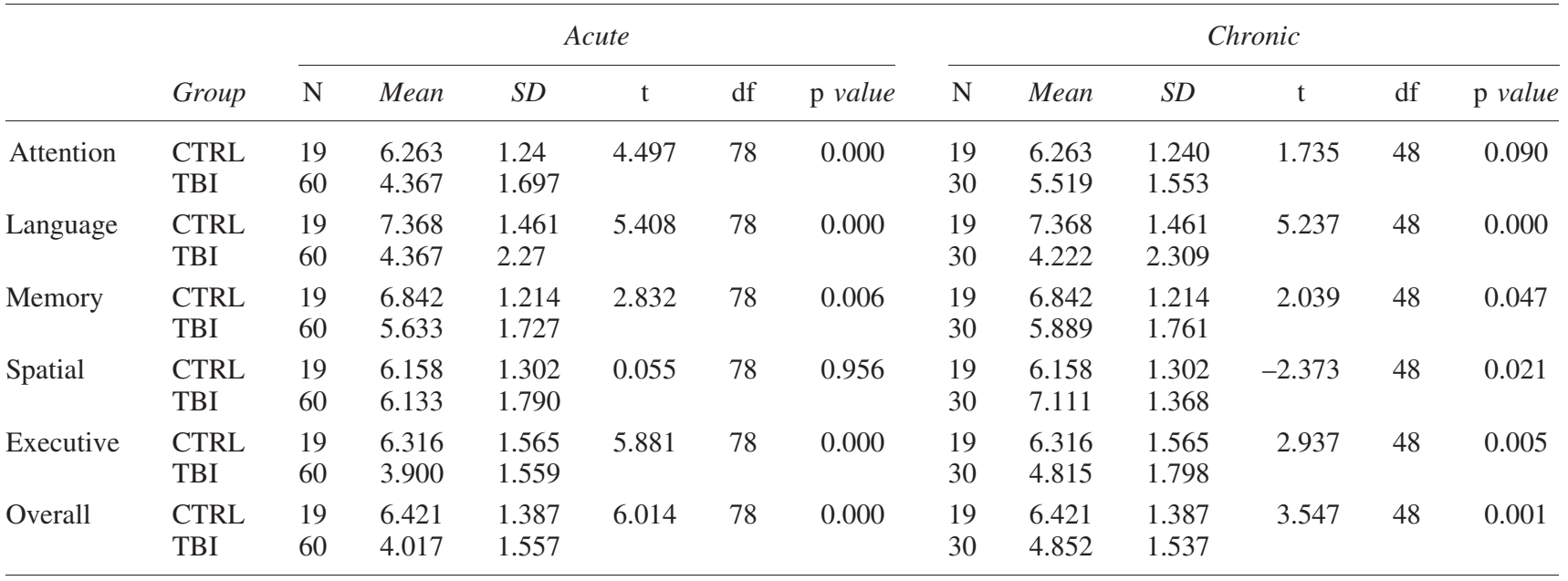

Interpretive category score legend: $1=$ severely impaired; $2=$ severe to moderately impaired; $3=$ moderately impaired; $4=$ mildly to moderately impaired; $5=$ mildly impaired; $6=$ below average; 7 =average; $8=$ above average; $9=$ superior; and $10=$ very superior.

S-NAB, Neuropsychological Assessment Battery-Screening; SD, standard deviation; CTRL, control; TBI, traumatic brain injury. 
Table 6a. Spearman's Rho Correlation Coefficient Table $(N=30)$ of Neuropsychological Performance Against Changes in FA, MD, and RD of the Various Brain Tracts Both at Acute and Chronic Phase

\begin{tabular}{|c|c|c|c|c|c|c|c|c|}
\hline \multirow[b]{2}{*}{ DTI metrics } & \multirow[b]{2}{*}{ White matter tracts of interest } & \multicolumn{3}{|c|}{ Acute } & \multicolumn{4}{|c|}{ Chronic } \\
\hline & & Attention & Language & Spatial & Attention & Language & Spatial & Executive \\
\hline \multirow[t]{7}{*}{ FA } & Middle cerebelar peduncle (acute) & & & & & $-0.440 *$ & & \\
\hline & Corona radiata (acute) & $-0.429 *$ & $-0.375^{*}$ & & $-0.441 *$ & $-0.415^{*}$ & & \\
\hline & Corona radiata (chronic) & & & & & $-0.400 *$ & & \\
\hline & Superior longitudinal fasiculus (acute) & & $-0.557^{\dagger}$ & & $-0.417 *$ & $-0.409 *$ & & \\
\hline & Cingulum (acute) & & & & & & $0.489 *$ & \\
\hline & Genu of corpus callosum (acute) & & $-0.443 *$ & & & & & \\
\hline & Splenium of corpus callosum (chronic) & & & & & & $0.402 *$ & \\
\hline \multirow[t]{3}{*}{ MD } & Superior longitudinal fasiculus (acute) & & & & $0.404 *$ & & & \\
\hline & Cingulum (acute) & & & & & & $-0.390^{*}$ & \\
\hline & Genu of corpus callosum (acute) & & & & & & $-0.404 *$ & \\
\hline \multirow[t]{10}{*}{$\mathrm{RD}$} & Middle cerebelar peduncle (acute) & & & & & $0.398 *$ & & \multirow{10}{*}{$0.389 *$} \\
\hline & Middle cerebelar peduncle (chronic) & & & & & $0.438 *$ & & \\
\hline & Corona radiata (acute) & $0.485^{*}$ & & & & $0.529^{\dagger}$ & & \\
\hline & Corona radiata (chronic) & & & & & & & \\
\hline & Superior longitudinal fasiculus (acute) & $0.487 *$ & & & $0.450^{*}$ & $0.491 *$ & & \\
\hline & Cingulum (acute) & & & & & $0.423^{*}$ & $-0.626^{\dagger}$ & \\
\hline & Cingulum (chronic) & & & & & & $-0.404^{*}$ & \\
\hline & Optic radiation (acute) & & & $-0.378 *$ & & & & \\
\hline & Genu of corpus callosum (acute) & & & & $0.378 *$ & & & \\
\hline & Splenium of corpus callosum (chronic) & & & & & & $-0.391 *$ & \\
\hline
\end{tabular}

*Correlation is significant at $p<0.05$ (two-tailed).

${ }^{\dagger}$ Correlation is significant at $p<0.01$ (two-tailed).

DTI, diffusion tensor imaging; FA, fractional anisotropy; MD, medial diffusivity; RD, radial diffusivity.

Table 6b. Spearman's Rho Correlation Coefficient Table $(N=19)$ of Neuropsychological Performance Against FA, MD, and RD of the Various Brain Tracts in Healthy Controls

\begin{tabular}{|c|c|c|c|c|c|c|c|c|}
\hline \multirow[b]{2}{*}{ Correlations } & \multirow[b]{2}{*}{ Group } & \multirow[b]{2}{*}{ Tracts vs. DTI parameters } & \multicolumn{6}{|c|}{ Cognitive domains } \\
\hline & & & Attention & Language & Memory & Spatial & Executive & Overall \\
\hline \multirow[t]{28}{*}{ Spearman's rho } & Control & Middle cerebellar peduncle_FA & -0.262 & -0.157 & -0.344 & -0.100 & 0.071 & -0.327 \\
\hline & & Corona radiata_FA & 0.000 & 0.282 & -0.301 & 0.000 & 0.000 & 0.065 \\
\hline & & Anterior limb of internal capsule_FA & 0.022 & 0.282 & -0.301 & 0.100 & 0.118 & -0.044 \\
\hline & & Posterior limb of internal capsule_FA & 0.022 & 0.031 & 0.172 & 0.020 & 0.212 & 0.218 \\
\hline & & Cingulum_FA & -0.196 & 0.000 & 0.129 & 0.299 & 0.071 & 0.000 \\
\hline & & Superior longitudinal fasiculus_FA1 & -0.240 & -0.063 & -0.172 & -0.080 & 0.000 & 0.087 \\
\hline & & Optic radiation_FA & -0.349 & 0.188 & -0.344 & -0.279 & -0.212 & -0.327 \\
\hline & & Genu of corpus callosum_FA & -0.109 & 0.344 & -0.129 & 0.139 & -0.212 & 0.218 \\
\hline & & Splenium of corpus callosum_FA & -0.065 & 0.282 & -0.344 & -0.040 & -0.094 & -0.240 \\
\hline & & Middle cerebellar peduncle_M̄M & -0.240 & -0.031 & 0.344 & -0.010 & -0.083 & 0.142 \\
\hline & & Corona radiata_MD & 0.175 & -0.282 & 0.086 & -0.219 & 0.012 & -0.131 \\
\hline & & Anterior limb of internal capsule_MD & 0.131 & 0.094 & 0.172 & -0.220 & -0.189 & 0.022 \\
\hline & & Posterior limb of internal capsule_MD & 0.186 & 0.220 & -0.302 & -0.060 & 0.047 & 0.197 \\
\hline & & Cingulum_MD & 0.000 & -0.141 & 0.345 & 0.040 & -0.035 & 0.218 \\
\hline & & Superior longitudinal fasiculus_MD & 0.372 & -0.125 & 0.000 & -0.060 & -0.236 & -0.109 \\
\hline & & Optic radiation_MD & 0.295 & -0.282 & 0.387 & 0.319 & 0.094 & 0.218 \\
\hline & & Genu of corpus callosum_MD & -0.022 & -0.282 & 0.387 & -0.020 & -0.047 & -0.131 \\
\hline & & Splenium of corpus callosum_MD & 0.164 & -0.031 & 0.345 & 0.249 & 0.283 & 0.426 \\
\hline & & Middle cerebellar peduncle_RD & 0.044 & 0.063 & 0.344 & 0.120 & -0.189 & 0.240 \\
\hline & & Corona radiata_RD & 0.131 & -0.251 & 0.301 & -0.040 & -0.035 & -0.022 \\
\hline & & Anterior limb of internal capsule_RD & 0.055 & -0.110 & 0.172 & -0.229 & -0.177 & -0.055 \\
\hline & & Posterior limb of internal capsule_RD & 0.175 & 0.125 & -0.302 & 0.060 & -0.260 & -0.131 \\
\hline & & Cingulum_RD & 0.109 & -0.141 & 0.129 & -0.179 & 0.035 & 0.131 \\
\hline & & Superior longitudinal fasiculus_RD & 0.284 & -0.094 & 0.043 & -0.020 & -0.024 & -0.153 \\
\hline & & Optic radiation_RD & 0.371 & -0.219 & 0.344 & 0.259 & 0.165 & 0.240 \\
\hline & & Genu of corpus callosum_RD & 0.044 & -0.376 & 0.258 & -0.100 & 0.047 & -0.262 \\
\hline & & Splenium of corpus callosum_RD & 0.044 & -0.235 & 0.345 & 0.060 & 0.165 & 0.251 \\
\hline & & $\mathrm{N}$ & 19 & 19 & 19 & 19 & 19 & 19 \\
\hline
\end{tabular}

DTI, diffusion tensor imaging; FA, fractional anisotropy; MD, medial diffusivity; RD, radial diffusivity. 
Table 7. Frequency Table of Neuropsychological Performance Status at 6 Months Post-Trauma in Comparison to Their Performance at Admission

\begin{tabular}{lrcrrr}
\hline & \multicolumn{5}{c}{ Neuropsychological performance status categories } \\
\cline { 2 - 4 } Domains & $1,1(\%)$ & $1,2(\%)$ & $2,1(\%)$ & $2,2(\%)$ & Fisher's exact \\
\hline Attention & $17(56.7)$ & $9(30.0)$ & $1(3.33)$ & $3(10.0)$ & 0.269 \\
Language & $19(63.3)$ & $8(26.7)$ & $1(3.33)$ & $2(6.67)$ & 0.234 \\
Memory & $10(33.3)$ & $4(13.3)$ & $7(26.7)$ & $16(53.3)$ & 0.253 \\
Spatial & $4(13.3)$ & $6(20.0)$ & $2(6.67)$ & $1(3.33)$ & 0.181 \\
Executive & $21(70.0)$ & $4(13.3)$ & $0(0.0)$ & $2(6.67)$ & 0.530 \\
Overall & $24(80.0)$ & & & 0.026 \\
\hline
\end{tabular}

Interpretive categories: impaired $=1$; not impaired $=2$.

procedure was completed within an average of $10 \mathrm{~h}$ post-trauma in order to identify the structural changes at a very early stage before they were subject to confounds, such as changes specific to environmental and recovery parameters. Subsequently, both a repeat imaging and neuropsychological assessment were performed at an average of 6 months post-trauma to characterize any pertinent changes over time.

Results showed associations between DTI and neuropsychological indices at both acute and chronic phases. Specifically, in the acute phase, the TBI group showed significantly poorer WM integrity across several tracts, including the splenium (SCC), PLIC, CG, and OR. Several other WM tracts beyond those initially affected also showed reduced FA at 6 months post-injury (i.e., CR, ALIC, SLF, and GCC). Interestingly, moderate effect sizes for changes in RD were observed from baseline to follow-up in the following regions: $\mathrm{CR}, \mathrm{CG}$, and $\mathrm{OR}$. Finally, with respect to neuropsychological functioning, patients with mTBI performed more poorly across all domains in the acute stage, when compared to healthy control participants. The majority of patients remained impaired with respect to executive functioning, and a sizable portion of the sample worsened over time on tasks of attention and language. Moreover, longitudinal analysis of WM tract changes, as they relate to cognition, showed several associations, indicating that reduced WM integrity was associated with specific neuropsychological decrements. Indeed, DTI indices in both acute and chronic stages related to cognition at both time points, and $\mathrm{RD}$ values in seven of the nine tracts studied was significantly associated with attention, language, spatial, and executive function in both phases of the study. Interestingly, no association was found between the domains of memory and any of the WM tracts investigated in this study.

Our findings of reduced FA, coupled with increased MD and RD in the acute phase, is considered indicative of vasogenic brain edema, which refers to the release of intracellular proteins into the brain parenchyma, also known as extracellular edema. ${ }^{18} \mathrm{An}$ increased $\mathrm{FA}_{\mathrm{a}}$, reduced $\mathrm{MD}_{\mathrm{a}}$, and $\mathrm{RD}_{\mathrm{a}}$ in the acute phase, on the other hand, may be indicative of cytotoxic edema, ${ }^{2,36}$ also known as intracellular edema. This type of cerebral edema in the early course of injury has been implicated in poor outcome, ${ }^{2,18}$ which could explain frequently observed neuropsychological impairment months or even years after the initial trauma event. The increased FA, MD, and rather an unchanged RD in the acute phase of this study is most likely indicative of reactive astrogliosis, which is a process where fibrous astrocytes migrate to the site of injury, locally increasing the density of the cells ${ }^{39}$ and diffusivity of the affected tissue. ${ }^{39,40}$

Vasogenic edema occurs in the acute phase, whereas demyelination occurs later. ${ }^{16}$ As such, even though the DTI markers for these pathogenic processes are the same, the timeframe of imaging changes rather indicate an ongoing vasogenic edema. These changes in DTI parameters were observed in the SCC, PLIC, CG, and OR (with statistically significant changes in most parameters). Other tracts, including the ALIC, SLF, and GCC, showed similar trends, although they did not reach statistical significance. Results showing minimally reduced $\mathrm{FA}_{\mathrm{a}}$ in the context of significantly increased MD of the SLF and GCC in the patient group, and its negative association with language function, are similar to those reported by Ingelese and colleagues ${ }^{33}$ and Arfanakis and colleagues. ${ }^{24}$ Cognitive impairment observed acutely in this group of patients coupled with the changing DTI metrics are likely immediate signs reflecting an ongoing edematous process, which is not observed by conventional CT or MRI.

In contrast, changes observed in $\mathrm{CR}_{\mathrm{a}}$ and $\mathrm{MCP}_{\mathrm{a}}$ were more suggestive of reactive astrogliosis and dovetails with findings recently reported by Croall and colleagues. ${ }^{41}$ Although some of these changes did not reach statistical significance, the effect size of 0.299 for the RD changes in CR, coupled with the effect size of 0.661 for the FA changes of $\mathrm{CR}$, for instance, does provide some moderate-to-large evidence of significant change in this tract. Higher FA value of the CR also negatively correlated with attention and language functions acutely. Its corresponding positive association with $\mathrm{RD}$ at baseline best explains the influence of reactive astrogliosis on cognition in the acute stage, as previously stated. ${ }^{40-}$ ${ }^{42}$ The importance of the above finding cannot be understated given that specific types of cerebral edema and gliosis in the acute phase have been implicated to negatively influence long-term neuropsychological performance in patients with mTBI. ${ }^{43-46}$

We also found that acute-phase DTI parameters in selected WM tracts were significantly associated with chronic domain-specific cognitive deficits. This includes those of the MCP, CR, SLF, CG, GCC, and SCC and their association with specific neurocognitive functions chronically, similar to findings of other studies. ${ }^{39-42,47,48}$ Miles and colleagues, for instance, noted that decreased FA values and increased MD values of the GCC, SCC, and PLIC during the acute phase were significantly related to executive dysfunction at 6 months follow-up. ${ }^{2,47}$ Kumar and colleagues and Matshushita and colleagues reported similar findings involving the GCC, SCC, and $\mathrm{NP}_{\mathrm{c}}$ in their studies of mild and moderate head injury. ${ }^{48,49}$ The mostly positive association between the unchanged or raised $\mathrm{RD}_{\mathrm{a}}$ of the MCP, CR, SLF, and CG with specific chronic deficits in attention, language, and spatial function likely reflect the long-term effects of acute vasogenic edema and reactive astrogliosis, a wellknown immune response in the immediate aftermath of central nervous system (CNS) injury. ${ }^{41}$ It is important to note that cerebral edema and cascading gliosis usually occur concurrently and are not necessarily independent of each other. 
Chronically, the continued alteration of DTI parameters, especially the $\mathrm{FA}_{\mathrm{c}}$ and $\mathrm{RD}_{\mathrm{c}}$, implies significant changes in WM integrity, where the myelin sheath or the axonal membrane or both may have been permanently damaged. ${ }^{19,20,36}$ This disruption may be irreversible, especially when a reduced $\mathrm{FA}_{\mathrm{c}}$ and elevated $\mathrm{MD}_{\mathrm{c}}$ and $\mathrm{RD}_{\mathrm{c}}$ are noted. ${ }^{2,19}$ Conversely, a reduced $\mathrm{FA}_{\mathrm{c}}$, unchanged $\mathrm{MD}$, and subtly altered $\mathrm{RD}_{\mathrm{c}}$ would mean that the microstructural changes to the WM are independent of gross tissue loss. ${ }^{43-46}$ The subtly altered $\mathrm{RD}_{\mathrm{c}}$ in this context would imply minor fiber damage without gross tissue loss. ${ }^{44}$ Such changes have also been implicated as a part of the dual effects of gliosis. ${ }^{46,50,51}$

FA measured at follow-up was significantly reduced, in comparison to the acute phase, with mostly unchanged $\mathrm{MD}_{\mathrm{c}}$ and subtly increased $\mathrm{RD}_{\mathrm{c}}$ across six tracts (i.e., $\mathrm{CR}$, ALIC, CG, SLF, OR, and GCC). Whereas the changes observed in the ALIC and OR are indicative of possible minor fiber damage and thus vasogenic edema, the change pattern observed in CR suggests irreversible consequences of astrogliosis. The mixed association between certain neurocognitive performances in the chronic stage (e.g., executive, spatial, and language function) and FA and RD of several tracts (i.e., MCP, CR, CG, and SCC), though possibly paradoxical, is not completely unexpected. Similar observations were found in a recent study with attribution to the multifaceted chronic phase findings. ${ }^{41}$ This included neuronal network reorganisation, ${ }^{52}$ spurting of axons with smaller calibres, ${ }^{53}$ glial scarring, ${ }^{46,50,51,54}$ effects of accumulated phosypholarated neurofilaments, ${ }^{55-59}$ and disruptive neurofibrillary tangles. ${ }^{60}$ Taken together, our findings further strengthen evidence for physiogenic influences on the prolonged functional and neuropsychological sequelae in patients with mTBI. ${ }^{61}$ Clearly, disruption of the connectomes (i.e., the connectivities between specific cortical and subcortical structures) by various neuropathological ${ }^{62,63}$ events posttrauma and the recovery process over time requires further study.

Interestingly, a common finding in our study was that the more severely affected fibers were generally the long tract fibers (SLF, $\mathrm{CR}$, and $\mathrm{CG}$ ) and commisural fibers (SCC and GCC). These fibers have an increased susceptibility to injury ${ }^{64}$ given their relatively long length and high membrane-to-cytoplasmic ratios. ${ }^{65,66}$ The corpus callosum, for example, as noted by Aoki and colleagues, is the major fiber bundle that enables communication between the hemispheres and is topographically organized. ${ }^{27,67}$ The CC in general is divided into the genu (GCC), the body, and the splenium (SCC). ${ }^{27}$ The CC has been long recognized as a frequently injured region owing to shear strain, ${ }^{68}$ and topographical location and external accelerational forces can seriously injure the fiber. ${ }^{69,70}$ The posterior region of the CC, namely, the SCC, often noted to be more vulnerable to injury than the anterior part (GCC), ${ }^{67,71-74}$ was replicated in this study as well; and the SCC was the only tract that showed statistically significant changes in all three DTI parameters at the acute stage.

Our findings lend support to the theory that microstructural changes occur within hours after the initial insult and affect the integrity of the WM, therefore leading to the varied manifestation of neuropsychological impairments, in agreement with some of the earlier studies reported in the literature. ${ }^{31,34,41,75,76}$ Strengths of our study include examination within a short timeframe from time of injury to imaging and neuropsychological testing within the acute phase, as well as consistent test-retest interval at 6-month followup. Although FA and MD have been shown to be very sensitive in detecting subtle WM changes that correlate with neuropsychological findings, one other parameter that has been included in this study, namely, $\mathrm{RD},{ }^{77}$ provides further insight into the presumed nature of the microstructural changes ${ }^{75,78,79}$ given that it is sensitive to myelin integrity $23,76,80$ and also other CNS immune response, such as gliosis. ${ }^{41}$ These parameters enabled us to detect the early deep WM microstructural changes, which might otherwise be confounded by ongoing secondary damage or recovery mechanisms. Additionally, the current study is unique in its longitudinal follow-up of DTI indices and cognitive functioning at 6 months post-trauma in a large cohort of well-characterized patients with relatively homogenous types of injuries. That said, findings may be limited owing to the significant heterogenity of the trauma observed in our sample as well as possible obscuring of certain differences owing to the nature of brain morphing and averaging, which is inherent in template-based analyses. Specifically, any deviation present in a small number of voxels within a region may be hidden by the averaging and therefore lead to low sensitivity to detect FA and MD changes. ${ }^{81,82}$

\section{Conclusion}

DTI is a useful technique used to assess the integrity of WM tracts in patients who have sustained mTBI. This study found significant correlations between neuropsychological deficits and WM tract integrity both within hours of neurotrauma and again 6 months later. These associations are likely attributable to alterations in the integrity of connectomes between specific cortical areas and subcortical structures, which is not evident in conventional MRI or CT procedures. Specific WM changes, as observed through the DTI parameters, especially in the acute period, and the corresponding neuropsychological impairments among the patients in the chronic phase, highlights the need to introduce appropriate imaging techniques early in patient management protocols for early prognostication and rehabilitative intervention. Further, our findings underscore the dynamic nature of brain injury and possible biological basis of cognitive dysfunction in the context of the postconcussive syndrome.

\section{Acknowledgments}

This research is partially funded by a University Malaya Research Grant (UMRG; RG008C-13HTM) and a High Impact Research Grant of University of Malaya (HIR-UM.C/625/1/HIRMOHE/12).

\section{Author Disclosure Statement}

No competing financial interests exist.

\section{References}

1. Faul, M., Xu, L., Wald, M.M., and Coronado, V.G. (2010). TBI in the United States: Emergency department visits, hospitalizations, and deaths 2002-2006. Centers for Disease Control and Prevention, National Center for Injury Prevention and Control: Atlanta, GA.

2. Shenton, M.E., Hamoda, H.M., Schneiderman, J.S., Bouix, S., Pasternak, O., Rathi, Y., Vu M.A., Purohit, M.P., Helmer, K., Koerte, I., Lin, A.P., Westin, C.F., Kikinis, R., Kubicki, M., Stern, RA., and Zafonte, R. (2012). A review of magnetic resonance imaging and diffusion tensor imaging findings in mild traumatic brain injury. Brain Imaging Behav. 6, 137-192.

3. Povlishock, J.T., Becker, D.P., Cheng, C.L., and Vaughan, G.W. (1983). Axonal change in minor head injury. J. Neuropathol. Exp. Neurol. 42, 225-242; as cited in: Büki, A., and Povlishock, J.T., (2006). All roads lead to disconnection?-Traumatic axonal injury revisited. Acta Neurochir. (Wien) 148, 181-194.

4. Bigler, E.D. (2010). Neuroimaging in mild traumatic brain injury. Psychol. Inj. Law 3, 36-49.

5. Bigler, E.D., and Bazarian, J.J. (2010). Diffusion tensor imaging: a biomarker for mild traumatic brain injury? Neurology 74, 626-627. 
6. Kou, Z., Wu, Z., Tong, K.A., Holshouser, B., Benson, R.R., Hu, J., and Haacke E.M. (2010). The role of advanced MR imaging findings as biomarkers of TBI. J. Head Trauma Rehabil. 25, 267-282.

7. Strangman, G.E., O’Niel-Pirozzi, T.M., Supelana, C., Goldstein, R. Katz, D.I., and Glenn, M.B. (2012). Fractional anisotropy helps predicts memory rehabilitation outcome after traumatic brain injury. NeuroRehabilitation 31, 295-310.

8. Kubicki, K.M., McCarley, R., Westin, C.F., Park, H.J., Maier, S., Kikinis, R., Jolesz, F.A., and Shenton, M.E. (2007). A review of diffusion tensor imaging studies in schizophrenia. J. Psychiatr. Res. 41, 15-30.

9. Sang, S.K., Sun, S.W., Ju, W.K., Lin, S.J., Cross, A.H., and Neufeld, A.H. (2003). Diffusion tensor imaging defects and differentiates axar and myelin degeneration in mouse optic nerve after retinal ischemia. NeuroImage 20, 1714-1722.

10. Budde, M.D., Xie, M., Cross, A.H., and Song, S.K. (2009). Axial diffusivity is the primary correlate of axonal injury in the experimental autoimmune encephalomyelitis spinal cord: a quantitative pixelwise analysis. J. Neurosci. 29, 2805-2813.

11. Song, S.K., Yoshino, J., Le, T.Q., Lin, S.J., Sun, S.W., Cross, A.H., and Armstrong, R.C. (2005). Demyelination increases radial diffusivity in corpus callosum of mouse brain. Neuroimage 26, 132-140.

12. Song, S.K., Sun, S.W., Ramsbottom, M.J., Chang, C., Russell, J., and Cross, A.H. (2002). Dysmyelination revealed through MRI as increased radial (but unchanged axial) diffusion of water. Neuroimage 17, 1429-1436.

13. Alexander, A.L., Hurley, S.A., Samsonov, A.A., Adluru, N., Hosseinbor, A.P., Mossahebi, P., Tromp do, P.M., Zakszewski, E., and Field, A.S. (2011). Characterization of cerebral white matter properties using quantitative magnetic resonance imaging stains. Brain Connect. 1, 423-446.

14. Green, R., Koshimori, Y., and Turner, G. (2010). Research Digest. Understanding the organic basis of persistent complaints in mTBI: findings from functional and structural neuroimaging. Neuropsychol. Rehabil. 20, 471-478.

15. Niogi, S.N., and Mukherjee, P. (2010). Diffusion tensor imaging of mild TBI. J. Head Trauma Rehabil. 25, 241-255.

16. Saatman, K.E. (2012). The complexity of traumatic axonal injury, in: Traumatic Brain and Spinal Cord Injury: Challenges and Developments. C. Morganti-Kossmann, R. Raghupathi, A. and Maas (eds) Traumatic Brain and Spinal Cord Injury: Challenges and Developments. Cambridge University Press: Cambridge, UK.

17. Belanger, H.G., Kretzmer, T., Yoash-Gantz, R., Pickett, T., and Tupler, L.A. (2009). Symptom complaints following combat-related traumatic brain injury: relationship to traumatic brain injury severity and posttraumatic stress disorder. J. Int. Neuropsychol. Soc. 16, 194 199.

18. Iffland II, P. H., Grant, G. A., and Janigro, D. (2014). Mechanisms of cerebral edema leading to early seizures after traumatic brain injury. In: Vascular Mechanisms in CNS Trauma. Springer: New York, pps. 29-45.

19. Grossman, E.J., Inglese, M., and Bammer, R. (2010). Mild traumatic brain injury: is diffusion imaging ready for primetime in forensic medicine? Top. Magn. Reson. Imaging 21, 379-386.

20. Schaefer, P.W., Grant, P.E., and Gonzalez, R.G. (2000). Diffusionweighted MR imaging of the brain. Radiology 217, 331-345.

21. Wilde, E.A., McCauley, S.R., Barnes, A., Wu, T.C., Chu, Z., Hunter, J.V., and Bigler, E.D. (2012). Serial measurement of memory and diffusion tensor imaging changes within the first week following uncomplicated mild traumatic brain injury. Brain Imaging Behav. 6, 319-328.

22. Farbota, K.D., Sodhi, A., Bendlin, B.B., McLaren, D.G., Xu, G., Rowley, H.A., and Johnson, S.C. (2012). Longitudinal volumetric changes following traumatic brain injury: a tensor-based morphometry study. J. Int. Neuropsychol. Soc. 18, 1006-1118.

23. Sidaros, A., Engberg, A.W., Sidaros, K., Liptrot, M.G., Herning, M., Petersen, P., Paulson, O.B., Jernigan, T.L., and Rostrup, E. (2008). Diffusion tensor imaging during recovery from severe traumatic brain injury and relation to clinical outcome: a longitudinal study. Brain 131, 559-572.

24. Arfanakis, K., Haughton, V.M., Carew, J.D., Rogers, B.P., Dempsey, R.J., and Meyerand, M.E. (2002). Diffusion tensor MR imaging in diffuse axonal injury. AJNR Am. J. Neuroradiol. 23, 794-802.

25. Hassan, K.M., Wilde, E.A., Miller, E.R., Patel, V.K., Staewen, T.D., Frisby, M.L., Garza, H.M., McCarthy, J.J., Hunter, J.V., and Levin,
H.S. (2014), Serial atlas-based diffusion tensor imaging study of uncomplicated mild traumatic brain injury in adults. J. Neurotrauma 31 , 466-475.

26. Perez, M.A., Adler, J., Kulkarni, N., Strain, J.F., Womack, K.B., Diaz Arrastia, R., and Marquez de la Plata, C.D. (2014). Longitudinal white matter changes after traumatic axonal injury. J. Neurotrauma 31, 1478-1485.

27. Aoki, Y., Inokuchi, R., Gunshin, M., Yahagi, N., and Suwa, H. (2012) Diffusion tensor imaging studies of mild traumatic brain injury: a meta-analysis. J. Neurol. Neurosurg. Psychiatry 83, 870-876.

28. Mukherjee, P., Miller, J.H., Shimony, J.S., Philip, J.V., Nehra, D., Snyder A.Z., Conturo, T.E., Neil, J.J., and McKinstry, R.C. (2002). Diffusion-tensor MR imaging of gray and white matter development during normal human brain maturation. AJNR Am. J. Neuroradiol. 23, 1445-1456.

29. Dodd A.B., Epstein K., Ling J.M., and Mayer A.R. (2014). Diffusion tensor imaging in semi-acute mild traumatic brain injury. J, Neurotrauma. 31, 1235-1248.

30. Nakayama, N., Okumura, A., Shinoda, J., Yasokawa, Y.T., Miwa, K., Yoshimura, S.I., and Iwama, T. (2006). Evidence for white matter disruption in traumatic brain injury without macroscopic lesions. J. Neurol. Neurosurg. Psychiatry 77, 850-855.

31. Henry, L.C., Tremblay, J., Tremblay, S., Lee, A., Brun, C., Lepore, N., Theoret, H., Ellemberg, D., and Lassonde, M. (2011). Acute and chronic changes in diffusivity measures after sports concussion. J. Neurotrauma 28, 2049-2059.

32. Niogi, S.N., Mukherjee, P., Ghajar, J., Johnson, C.E., Kolster, R., Lee, H., Suh, M., Zimmerman, R.D., Manley, G.T., and McCandliss, B.D (2008). Structural dissociation of attentional control and memory in adults with and without mild traumatic brain injury. Brain 131, 3209 3221 .

33. Inglese, M., Makani, S., Johnson, G., Cohen, B.A., Silver, J.A., Gonen, O., and Grossman, R.I. (2005). Diffuse axonal injury in mild traumatic brain injury: a diffusion tensor imaging study. J. Neurosurg. 103, 298-303.

34. Toth, A., Kovacs, N., Perlaki, G., Orsi, G., Aradi, M., Komaromy, H. Ezer, E., Bukovics, P., Farkas, O., Janszky, J., Doczi, T., Buki, A., and Schwarcz, A. (2013). Multi-modal magnetic resonance imaging in the acute and sub-acute phase of mild traumatic brain injury: can we see the difference? J. Neurotrauma 30, 2-10.

35. Kraus, M.F., Susmaras, T., Caughlin. B.P., Walker, C.J., Sweeney, J.A., and Little, D.M. (2007). White matter integrity and cognition in chronic traumatic brain injury: a diffusion tensor imaging study. Brain 130, 2508-2519.

36. Bazarian, J.J., Zhong, J., Blyth, B., Zhu, T., Kavcic, V., and Peterson, D. (2007). Diffusion tensor imaging detects clinically important axonal damage after mild traumatic brain injury: a pilot study. J. Neurotrauma 24, 1447-1459.

37. Smith, S.M., Jenkinson, M., Johansen-Berg, H., Rueckert, D., Nichols, T.E., Mackay, C.E., Watkins, K.E., Ciccarelli, O., Cader, M.Z., Matthews, P.M., and Behrens, T.E. (2006). Tract-based spatial statistics: voxelwise analysis of multi-subject diffusion data. Neuroimage 31, 1487-1505

38. Stern, R.A., and White, T. (2003). Neuropsychological Assessment Battery. Psychological Assessment Resources: Lutz, FL.

39. Pekny, M., and Nilsson, M. (2005). Astrocyte activation and reactive gliosis. Glia 50, 427-434.

40. Budde, M.D., Janes, L., Gold, E., Turtzo, L.C., and Frank, J.A. (2011). The contribution of gliosis to diffusion tensor anisotropy and tractography following traumatic brain injury: validation in the rat using Fourier analysis of stained tissue sections. Brain 134, 2248-2260.

41. Croall, I.D., Cowie, C.J., He, J., Peel, A., Wood, J., Aribisala, B.S., Mitchell, P., Mendelow, A.D., Smith, F.E., Millar, D., Kelly, T., and Blamire, A.M. (2014). White matter correlates of cognitive dysfunction after mild traumatic brain injury. Neurology 83, 494-501.

42. Chen, Y., and Swanson, R.A. (2003). Astrocytes and brain injury. J. Cereb. Blood Flow Metab. 23, 137-149.

43. Gold, B.T., Powell, D.K., Andersen, A.H., and Smith, C.D. (2010) Alterations in multiple measures of white matter integrity in normal women at high risk for Alzheimer's disease. Neuroimage 52, 1487 1494

44. Burzynska, A.Z., Preuschhof, C., Backman, L., Nyberg, L., Li, S.C., Lindenberger, U., and Heekeren, H.R. (2010) Age related differences in white matter microstructure: region-specific patterns of diffusivity. Neuroimage 49, 2104-2112. 
45. Sen, P.N., and Basser, P.J. (2005). A model for diffusion in white matter in the brain. Biophys. J. 89, 2927-2938.

46. Rovaris, M., Gass, A., Bammer, R., Hickman, S.J., Ciccarelli, O., Miller, D.H., and Filippi, M. (2005). Diffusion MRI in multiple sclerosis. Neurology 65, 1526-1532.

47. Miles, L., Grossman, R.I., Johnson, G., Babb, J.S., Diller, L., and Inglese, M. (2008). Short-term DTI predictors of cognitive dysfunction in mild traumatic brain injury. Brain Inj. 22, 115-122.

48. Kumar, R., Gupta, R.K., Husain, M., Chaudhry, C., Srivastava, A., Saksena, S., and Rathore, R.K. (2009). Comparative evaluation of corpus callosum DTI metrics in acute mild and moderate traumatic brain injury: its correlation with neuropsychometric tests. Brain Inj. 23, 675-685.

49. Matsushita, M., Hosoda, K., Naitoh, Y., Yamashita, H., and Kohmura, E. (2011). Utility of diffusion tensor imaging in the acute stage of mild to moderate traumatic brain injury for detecting white matter lesions and predicting long-term cognitive function in adults. J. Neurosurg. $115,130-139$.

50. Werring, D.J., Clark, C.A., Barker, G.J., Thompson, A.J., and Miller, D.H. (1999). Diffusion tensor imaging of lesions and normal-appearing white matter in multiple sclerosis. Neurology 52, 1626-1632.

51. Pekny, M., Wilhelmsson, U., and Pekna, M. (2014). The dual role of astrocyte activation and reactive gliosis. Neurosci. Lett. 565, 30-38.

52. Voets, N.L., Adcock, J.E., Flitney, D.E., Behrens, T.E. J., Hart, Y., Stacey, R., Carpenter, K., and Matthews, P.M. (2006). Distinct right frontal lobe activation in language processing following left hemisphere injury. Brain 129, 754-766.

53. Jafari, S.S., Maxwell, W.L., Neilson, M., and Graham, D.I. (1997). Axonal cytoskeletal changes after non-disruptive axonal injury. J. Neurocytol. 26, 207-221.

54. Stichel, C.C., and Müller, H.W. (1998). The CNS lesion scar: new vistas on an old regeneration barrier. Cell Tissue Res. 294, 1-9.

55. Stein, T.D., Alvarez, V.E., and McKee, A.C. (2014). Chronic traumatic encephalopathy: a spectrum of neuropathological changes following repetitive brain trauma in athletes and military personnel. Alzheimers Res. Ther. 6, 4

56. Buki, A., and Povlishock, J.T. (2006). All roads lead to disconnection? Traumatic axonal injury revisited. Acta Neurochirurg. 148, 181-194.

57. Strong, M.J., Strong, W.L., Jaffe, H., Traggert, B., Sopper, M.M., and Pant, H.C. (2001). Phosphorylation state of the native high molecular weight neurofilament subunit protein from cervical spinal cord in sporadic amyotrophic lateral sclerosis. J. Neurochem. 76, 1315-1325.

58. Ghonemi, M.O., Rabah, A.A., Saber, H.M., and Radwan, W. (2013). Role of Phosphorylated Neurofilament $\mathrm{H}$ as a diagnostic and prognostic marker in traumatic brain injury. Egyptian J. Crit. Care Med. 1, 139-144.

59. Saljo, A., Bao, F., Haglid, K.G., and Hansson, H.A. (2000). Blast exposure causes redistribution of phosphorylated neurofilament subunits in neurons of the adult rat brain. J. Neurotrauma 17, 719-726.

60. Blennow, K., Hardy, J., and Zetterberg, H. (2012). The neuropathology and neurobiology of traumatic brain injury. Neuron 76, 886-899.

61. Wada, T., Asano, Y., and Shinoda, J. (2012). Decreased fractional anisotropy evaluated using tract-based spatial statistics and correlated with cognitive dysfunction in patients with mild traumatic brain injury in the chronic stage. AJNR Am. J. Neuroradiol. 33, 2117-2122.

62. Patt, S., and Brodhun, M. (1999). Neuropathological sequelae of traumatic injury in the brain. An overview. Exp. Toxicol. Pathol. 51, 119-123.

63. Bigler, E.D. (2003). Neurobiology and neuropathology underlie the neuropsychological deficits associated with traumatic brain injury. Arch. Clin. Neuropsychol. 18, 595-621.

64. Bigler, E.D., and Maxwell, W.L. (2012). Neuropathology of mild traumatic brain injury: relationship to neuroimaging findings. Brain Imaging Behav. 6, 108-136.

65. Korn, A., Golan, H., Melamed, I., Pascual-Marqui, R., and Friedman, A. (2005). Focal cortical dysfunction and blood-brain barrier disruption in patients with postconcussion syndrome. J. Clin. Neurophysiol. 22, 1-9.

66. McKee, A.C., and Robinson, M.E. (2014). Military-related traumatic brain injury and neurodegeneration. Alzheimers Dement. 10, S242-S253.

67. Gennarelli, T.A., Thibault, L.E., Adams, J.H., Graham, D.I., Thompson, C.J., and Marcincin, R.P. (1982). Diffuse axonal injury and traumatic coma in the primate. Ann. Neurol. 12, 564-574.
68. Gentry, L.R., Godersky, J.C., and Thompson, B. (1988). MR imaging of head trauma: review of the distribution and radiopathologic features of traumatic lesions. Am. J. Roentgenol. 150, 663-672.

69. Chan, Y.L., Chu, W.C., Wong, G.W., and Yeung, D.K. (2003). Diffusion-weighted MRI in shaken baby syndrome. Pediatr. Radiol. 33, 574-577.

70. Bonnier, C., Mesples, B., and Gressens, P. (2004). Animal models of shaken baby syndrome: revisiting the pathophysiology of this devastating injury. Pediatr. Rehabil. 7, 165-171.

71. Wang, J.Y., Bakhadirov, K., Devous, M.D., Abdi, H., McColl, R., Moore, C., Marquez de la Plata, Ding, K., Whittemore, A., Babcock, E., Rickbeil, T., Dobervich, J., Kroll, D., Dao, B., Mohindra, N., Madden, C.J., and Diaz-Arrastia, R. (2008). Diffusion tensor tractography of traumatic diffuse axonal injury. Arch. Neurol. 65, 619-626.

72. Takaoka, M., Tabuse, H., Kumura, E., Nakajima, S., Tsuzuki, T., Nakamura, K., Okada, A., and Sugimoto, H. (2002). Semiquantitative analysis of corpus callosum injury using magnetic resonance imaging indicates clinical severity in patients with diffuse axonal injury. J. Neurol. Neurosurg. Psychiatry 73, 289-293.

73. Shiramizu, H., Masuko, A., Ishizaka, H., Shibata, M., Atsumi, H. Imai, M., Osada, T., Mizokami, Y., Baba, T., and Matsumae, M. (2008). Mechanism of injury to the corpus callosum, with particular reference to the anatomical relationship between site of injury and adjacent brain structures. Neurol. Med. Chir. (Tokyo) 48, 1-7; discussion, 6-7.

74. Imperati, D., Colcombe, S., Kelly, C., Di Martino, A., Zhou, J., Castellanos, F.X., and Milham, M.P. (2011). Differential development of human brain white matter tracts. PLoS ONE 6; 234-237.

75. Newcombe, V.F., Williams, G.B., Nortje, J., Bradley, P.G., Harding, S.G., Smielewski, P., Coles, J.P., Maiya, B., Gillard, J.H., Hutchinson, P.J., Pickard, J.D., Carpenter, T.A., and Menon, D.K. (2007). Analysis of acute traumatic axonal injury using diffusion tensor imaging. Br. J. Neurosurg. 21, 340-348.

76. Mac Donald, C.L., Dikranian, K., Bayly, P., Holtzman, D., and Brody, D. (2007). Diffusion tensor imaging reliably detects experimental traumatic axonal injury and indicates approximate time of injury. J. Neurosci. 27, 11869-11876.

77. Zhuo, J., Xu, S., Proctor, J. L., Mullins, R. J., Simon, J. Z., Fiskum, G., and Gullapalli, R. P. (2012). Diffusion kurtosis as an in vivo imaging marker for reactive astrogliosis in traumatic brain injury. Neuroimage 59, 467-477.

78. Alexander, A.L., Lee, J.E., Lazar, M., and Field, A.S. (2007). Diffusion tensor imaging of the brain. Neurotherapeutics 4, 316-329.

79. Chu, Z., Wilde, E.A., Hunter, J.V., McCauley, S.R., Bigler, E.D., Troyanskaya, M., Yallampalli, R., Chia, J.M., and Levin, H.S. (2010). Voxel-based analysis of diffusion tensor imaging in mild traumatic brain injury in adolescents. AJNR Am. J. Neuroradiol. 31, 340-346.

80. Sang, S.K., Sun, S.W., Ju, W.K., Lin, S.J., Cross, A.H., and Neufeld, A.H. (2003). Diffusion tensor imaging defects and differentiates axar and myelin degeneration in mouse optic nerve after retinal ischemia. NeuroImage 20, 1714-1722.

81. Bigler, E.D. (2013). Neuroimaging biomarkers in mild traumatic brain injury (mTBI). Neuropsychol. Rev. 23, 169-209.

82. Bach, M., Laun, F.B., Leemans, A., Tax, C.M., Biessels, G.J., Stieltjes, B., and Maier-Hein, K.H. (2014). Methodological considerations on tract-based spatial statistics (TBSS). Neuroimage 100, 358369 .

Address correspondence to: Vigneswaran Veeramuthu, MEdPsych Division of Neurosurgery Department of Surgery University of Malaya 50603 Kuala Lumpur Wilayah Persekutuan Malaysia

E-mail: vicveera@gmail.com 